\title{
Energy inequalities for cutoff functions and some applications
}

\author{
Sebastian Andres*, Martin T. Barlow ${ }^{\dagger}$
}

\begin{abstract}
Let $(\mathcal{X}, d, m)$ be a metric measure space with a local regular Dirichlet form. We establish necessary and sufficient conditions for upper heat kernel bounds with subdiffusive space-time exponent to hold. This characterization is stable under rough isometries, that is it is preserved under bounded perturbations of the Dirichlet form. Further, we give a criterion for stochastic completeness in terms of a Sobolev inequality for cutoff functions. As an example we show that this criterion applies to an anomalous diffusion on a geodesically incomplete fractal space, where the well-established criterion in terms of volume growth fails.

Keywords: Heat kernels, Sobolev inequality, Faber-Krahn inequality, rough isometry, stochastic completeness, conservativeness, Sierpinski carpet, anomalous diffusion
\end{abstract}

Subject Classification. Primary: 60J35; Secondary: 60J25, 31C05, 31C25

\section{Introduction}

Let $(\mathcal{X}, d)$ be a locally compact metric space and let $m$ be a positive Radon measure on $\mathcal{X}$ with $\operatorname{supp}[m]=\mathcal{X}$. We will refer to such a triple $(\mathcal{X}, d, m)$ as a metric measure space, and denote by $\langle.,$.$\rangle the inner product in L^{2}(\mathcal{X}, m)$. We consider a regular, strongly local Dirichlet form $(\mathcal{E}, \mathcal{F})$ on $L^{2}(\mathcal{X}, m)$ (see [FOT]). Let $\mathcal{L}$ be the (negative definite) generator of $\mathcal{E}$; this is a self-adjoint operator in $L^{2}(\mathcal{X}, m)$ such that

$$
\mathcal{E}(f, g)=-\langle\mathcal{L} f, g\rangle \quad \text { for all } f \in \mathcal{D}(\mathcal{L}), g \in \mathcal{F},
$$

and let $\left\{P_{t}\right\}_{t \geq 0}$ be the associated semigroup. If $P_{t}$ has a density $p_{t}(x, y)$ with respect to $m$ then after some regularization we call this the heat kernel on the metric measure Dirichlet space (or $M M D$ space) $(\mathcal{X}, d, m, \mathcal{E})$. Our main interest is in upper bounds on $p_{t}(x, y)$. Write $B(x, r)$ for balls in $(\mathcal{X}, d)$ and set

$$
V(x, r)=m(B(x, r)) .
$$

\footnotetext{
* Research partially supported by NSERC (Canada)
}

${ }^{\dagger}$ Research partially supported by NSERC (Canada) and by Trinity College, Cambridge 
Most familiar are Gaussian upper bounds of the form

$$
p_{t}(x, y) \leq \frac{c_{1}}{V\left(x, t^{1 / 2}\right)} \exp \left(-c_{2} \frac{d(x, y)^{2}}{t}\right)
$$

these arise (with lower bounds of the same form but with different constants) in the case of uniformly elliptic divergence form PDE, and manifolds with Ricci curvature bounded uniformly below - see $\mathrm{Ar}, \mathrm{LY}]$.

If (1.2) holds we will say $(\mathcal{X}, \mathcal{E})$ satisfies the condition $\operatorname{UHK}(2)$; if in addition Gaussian lower bounds hold we say HK(2) holds. One can ask for characterizations of these bounds, and in particular for characterizations which are stable, that is that are preserved under bounded perturbation of the Dirichlet form. More precisely, a property $(\mathrm{P})$ of $(\mathcal{X}, \mathcal{E})$ is stable if when $\left(\mathcal{E}_{i}, \mathcal{F}\right)$ are two Dirichlet forms on $L^{2}(\mathcal{X}, m)$ with

$$
C^{-1} \mathcal{E}_{1}(f, f) \leq \mathcal{E}_{2}(f, f) \leq C \mathcal{E}_{1}(f, f), \quad f \in \mathcal{F},
$$

then $(P)$ holds for $\left(\mathcal{X}, \mathcal{E}_{1}\right)$ if and only if it holds for $\left(\mathcal{X}, \mathcal{E}_{2}\right)$. In the manifold case stability for $\operatorname{HK}(2)$ was proved in [Gr0, SC1] by showing that these Gaussian bounds are equivalent to volume doubling (denoted VD) plus a family of Poincaré inequalities - see below for the precise definitions. If $\mathrm{VD}$ holds then stability for $\mathrm{UHK}(2)$ is a consequence of the results of [Gr3], where it is shown that $\operatorname{UHK}(2)$ is equivalent to a Faber Krahn inequality FK(2), which controls the smallest eigenvalue of domains in $\mathcal{X}$.

The Gaussian bounds (1.2) arise due to the standard space-time scaling relation $t=r^{2}$. More general possibilities can arise; for various exact fractals (see [Ba1]) one can have $V(x, r) \asymp r^{\alpha}$ and a space time scaling of $t=r^{\beta}$, where $\alpha \in[1, \infty)$ and $\beta \in[2,1+\alpha]$; the case when $\beta \neq 2$ is called anomalous diffusion. Since we wish to be able to consider spaces with different local and global structure, we introduce a more general space-time scaling function $\Psi$. Let $\beta_{L} \geq 2, \beta \geq 2$, and set

$$
\Psi(r)=\Psi_{\beta_{L}, \beta}(r)= \begin{cases}r^{\beta_{L}} & \text { if } 0 \leq r \leq 1 \\ r^{\beta} & \text { if } r>1\end{cases}
$$

We will write $\operatorname{UHK}(\Psi)$ for the heat kernel upper bounds associated with $\Psi$ - see Definition 1.6 below for their precise form. Our main theorem is a stable characterization of UHK $(\Psi)$, in terms of Faber-Krahn inequality $\mathrm{FK}(\Psi)$, and a new condition denoted $\operatorname{CSA}(\Psi)$, which controls the energy of cutoff functions in annuli.

To state our results precisely, we need a number of further definitions.

Since $\mathcal{E}$ is regular, each function $f \in \mathcal{F}$ admits a quasi-continuous version $\tilde{f}$ (see Theorem 2.1.3 in [FOT]). Throughout the paper, we will abuse notation and take the quasi-continuous version of $f$ without writing $\tilde{f}$. Another consequence of regularity is that $\mathcal{E}(f, g)$ can be written in terms of a signed measure $\Gamma(f, g)$ as

$$
\mathcal{E}(f, g)=\int_{\mathcal{X}} d \Gamma(f, g)
$$


For any essentially bounded $f \in \mathcal{F}, \Gamma(f, f)$ is the unique Borel measure on $\mathcal{X}$ (called the energy measure) on $\mathcal{X}$ satisfying

$$
\int_{\mathcal{X}} g d \Gamma(f, f)=2 \mathcal{E}(f, f g)-\mathcal{E}\left(f^{2}, g\right)
$$

for all essentially bounded $g \in \mathcal{F} ; \Gamma(f, g)$ is then defined by polarization.

Example. (Davies $[\mathrm{D}]$ ). Let $(M, d)$ be a manifold with Riemannian volume measure $\mu$, and $\mathcal{E}(f, f)=\int|\nabla f|^{2} d \mu$. Let $\sigma>0$, and $d m=\sigma^{2} d \mu$. Then

$$
\mathcal{L} f=\sigma^{-2} \nabla\left(\sigma^{2} \nabla f\right),
$$

and $d \Gamma(f, f)=|\nabla f|^{2} \sigma^{2} d \mu$.

For later use we collect from [FOT, Section 3.2] some properties of the energy measure.

i) Locality. For all functions $f, g \in \mathcal{F}$ and all measurable sets $G \subset \mathcal{X}$ on which $f$ is constant

$$
\mathbb{1}_{G} d \Gamma(f, g)=0 .
$$

ii) Leibniz and chain rules. For $f, g \in \mathcal{F}$ essentially bounded and $\varphi \in C^{1}(\mathbb{R})$,

$$
\begin{aligned}
d \Gamma(f g, h) & =f d \Gamma(g, h)+g d \Gamma(f, h), \\
d \Gamma(\varphi(f), g) & =\varphi^{\prime}(f) d \Gamma(f, g) .
\end{aligned}
$$

We note also the following result of Le Jan [LJ, Proposition 1.5.5(b)] - see also [Mos], p. 389 for a simple proof.

Lemma 1.1. Let $\mathcal{X}$ be a $M M D$ space. Suppose that $\left(\mathcal{E}_{i}, \mathcal{F}\right), i=1,2$, are strongly local regular Dirichlet forms that satisfy

$$
C^{-1} \mathcal{E}_{1}(f, f) \leq \mathcal{E}_{2}(f, f) \leq C \mathcal{E}_{1}(f, f), \quad f \in \mathcal{F} .
$$

Then their energy measures $\Gamma^{(i)}$ satisfy

$$
C^{-1} d \Gamma^{(1)}(f, f) \leq d \Gamma^{(2)}(f, f) \leq C d \Gamma^{(1)}(f, f), \text { for all } f \in \mathcal{F} .
$$

We now introduce a number of conditions which the space $\mathcal{X}$ and Dirichlet form $\mathcal{E}$ may or may not satisfy.

Definition 1.2. We say that $(\mathcal{X}, d, m)$ satisfies volume doubling (VD) if there exists a constant $C_{D}$ such that for every $x \in \mathcal{X}, r>0$,

$$
V(x, 2 r) \leq C_{D} V(x, r) .
$$

We next introduce the Faber-Krahn inequality: see [GT], Section 3.3 for more details. For any open set $D \subset \mathcal{X}, \mathcal{F}_{D}$ is defined to be the closure in $\mathcal{F}$ of the set of all functions in $\mathcal{F}$ that are compactly supported in $D$. For $D \subset \mathcal{X}$ we write $\lambda_{1}(D)$ for the smallest (Dirichlet) eigenvalue of $\mathcal{L}$ on $D$; this can be defined by the variational formula

$$
\lambda_{1}(D)=\inf \left\{\frac{\mathcal{E}(f, f)}{\|f\|_{2}^{2}}: f \in \mathcal{F}_{D}, f \neq 0\right\} .
$$


Definition 1.3. The MMD space $(\mathcal{X}, \mathcal{E})$ satisfies the Faber-Krahn inequality $\mathrm{FK}(\Psi)$ if there exists a constant $C_{F}$ and $\nu>0$ such that for any ball $B=B(x, r)$ and open set $D \subset B$

$$
\lambda_{1}(D) \geq \frac{C_{F}}{\Psi(r)}(m(B) / m(D))^{\nu} .
$$

We remark that the value of $\nu$ turns out to be unimportant.

Definition 1.4. We say that the Poincaré inequality $\mathrm{PI}(\Psi)$ holds if there exists a constant $C_{P}$ such that for all balls $B=B(x, r)$ and $f \in \mathcal{F}$,

$$
\inf _{a \in \mathbb{R}} \int_{B}(f-a)^{2} d m=\int_{B}\left(f-\bar{f}_{B}\right)^{2} d m \leq C_{P} \Psi(r) \int_{B} d \Gamma(f, f) .
$$

Here $\bar{f}_{B}$ is the mean of $f$ on $B$.

Associated with the Dirichlet form $(\mathcal{E}, \mathcal{F})$ and semigroup $\left(P_{t}\right)$ is a Hunt process $X=$ $\left(X_{t}, t \geq 0, \mathbb{P}^{x}, x \in \mathcal{X}-\mathcal{N}\right)$. Here $\mathcal{N}$ is 'properly exceptional': $m(\mathcal{N})=0$ and $\mathbb{P}^{x}\left(X_{t} \in\right.$ $\mathcal{N}$ for some $t>0)=0$ for all $x \in \mathcal{X}-\mathcal{N}$ - see [FOT, p. 134]. This Hunt process is unique up to a properly exceptional set - see [FOT, Theorem 4.2.7]. We fix $X$ and $\mathcal{N}$, and write

$$
\mathcal{X}_{0}=\mathcal{X}-\mathcal{N}
$$

While the semigroup $\left(P_{t}\right)$ associated with $\mathcal{E}$ is defined on $L^{2}$, a more precise version, with better regularity properties, can be obtained if we set, for bounded Borel $f$,

$$
P_{t} f(x)=\mathbb{E}^{x} f\left(X_{t}\right), \quad x \in \mathcal{X}_{0}
$$

The heat kernel associated with $\left(P_{t}\right)$ (if it exists) is a measurable function $p_{t}(x, y)$ : $(0, \infty) \times \mathcal{X}_{0} \times \mathcal{X}_{0} \rightarrow(0, \infty)$ such that

$$
\begin{aligned}
\mathbb{E}^{x} f\left(X_{t}\right) & =P_{t} f(x)=\int p_{t}(x, y) f(y) m(d y), x \in \mathcal{X}_{0}, f \in L^{\infty}(\mathcal{X}), \\
p_{t}(x, y) & =p_{t}(y, x), \text { for all } t>0, x, y \in \mathcal{X}_{0}, \\
p_{s+t}(x, z) & =\int p_{s}(x, y) p_{t}(y, z) m(d y), \text { for all } s>0, t>0, x, z \in \mathcal{X}_{0} .
\end{aligned}
$$

While (1.10) only defines $p_{t}(x, \cdot) m$-a.e., using the Chapman-Kolmogorov equation (1.12) one can regularise $p_{t}(x, y)$ so that (1.10) $-(1.12)$ hold on all of $\mathcal{X}_{0}$. For more details see GT].

Define the function

$$
\Phi(R, t)=\sup _{s>0}\left(\frac{R}{s}-\frac{t}{\Psi(s)}\right) .
$$

The following lemma summarises some properties of this function - see Section 3.3 of [GT] and in particular Example 3.18. 
Lemma 1.5. $\Phi(R, t)$ is non-negative, increasing in $R$ and decreasing in $t$. We have

$$
\Phi(R, t) \asymp \begin{cases}\left(\frac{R^{\beta} L}{t}\right)^{1 /\left(\beta_{L}-1\right)}, & \text { if } t \leq R, \\ \left(\frac{R^{\beta}}{t}\right)^{1 /(\beta-1)}, & \text { if } t \geq R .\end{cases}
$$

Further $\Phi(R, \Psi(R)) \leq \beta_{2}^{-1 /\left(\beta_{2}-1\right)}$, where $\beta_{2}=\beta_{L} \vee \beta$.

We define $\Psi^{-1}$ to be the inverse of $\Psi$, so that

$$
\Psi^{-1}(s)=s^{1 / \beta_{L}} 1_{(s \leq 1)}+s^{1 / \beta} 1_{(s>1)} .
$$

Definition 1.6. We say $\left(p_{t}\right)$ satisfies $\operatorname{UHK}(\Psi)$ if there exists a properly exceptional set $\mathcal{N}_{0}$ and constants $c_{1}, c_{2}$ such that

$$
p_{t}(x, y) \leq V\left(x, \Psi^{-1}\left(c_{1} t\right)\right)^{-1} \exp \left(-\Phi\left(c_{2} d(x, y), t\right)\right)
$$

for all $t>0$ and for all $x, y \in \mathcal{X}-\mathcal{N}_{0}$. If a similar lower bound (with different constants $c_{i}$ ) also holds then we say that $\operatorname{HK}(\Psi)$ holds.

When $\Psi(r)=r^{\beta}$ we will write $\operatorname{PI}(\beta)$ etc. for the condition $\operatorname{PI}(\Psi)$.

As explained above, we wish to find a stable characterization of the heat kernel bounds $\operatorname{UHK}(\Psi)$. In view of Lemma 1.1, the characterizations of $\operatorname{HK}(2)$ and $\operatorname{UHK}(2)$ in terms of Faber-Krahn and Poincaré inequalities are stable. It is easy to see that the natural generalization of these to more general $\Psi$ fails. Let $\Psi_{2} \geq \Psi_{1}$ with $\Psi_{2}(r) / \Psi_{1}(r) \rightarrow \infty$, and let $\mathcal{X}$ be an unbounded space satisfying $\operatorname{HK}\left(\Psi_{1}\right)$. Then $\mathcal{X}$ also satisfies $\operatorname{FK}\left(\Psi_{1}\right)$ and $\operatorname{PI}\left(\Psi_{1}\right)$, and so by the monotonicity of these conditions in $\Psi$, it is immediate that $\mathcal{X}$ satisfies $\operatorname{FK}\left(\Psi_{2}\right)$ and $\operatorname{PI}\left(\Psi_{2}\right)$. However, it is straightforward to check that $\operatorname{HK}\left(\Psi_{1}\right)$ and $\operatorname{UHK}\left(\Psi_{2}\right)$ cannot both hold. At a more fundamental level, the conditions $\operatorname{PI}(\Psi)$ and $\mathrm{FK}(\Psi)$ ensure that the heat equation homogenises over a ball of radius $R$ in time at most $\Psi(R)$, but do not exclude the possibility that this might occur more quickly. To 'capture' $\mathrm{HK}(\Psi)$ one needs a condition which gives an upper bound on the rate at which heat, or the diffusion $X$, can move on the space $\mathcal{X}$. Such a condition was found in [BB3, BBK], which gave a stable characterization of $\operatorname{HK}(\Psi)$.

Definition 1.7. Let $U \subset V$ be open sets in $\mathcal{X}$ with $U \subset \bar{U} \subset V$. We say a continuous function $\varphi$ is a cutoff function for $U \subset V$ if $\varphi=1$ on $U$ and $\varphi=0$ on $V^{c}$.

Definition 1.8 (Condition $(\mathrm{CS}(\Psi))$. (See [BB3, $\overline{\mathrm{BBK}}]$.) We say that condition $\mathrm{CS}(\Psi)$ holds if there exist constants $c_{1}$ and $\theta \in(0,1]$ such that the following holds. For every ball $B\left(x_{0}, r\right)$ there exists a cutoff function $\varphi$ with $\varphi=1$ on $B\left(x_{0}, r / 2\right)$ and $\varphi=0$ on $B\left(x_{0}, r\right)^{c}$, with the following properties.

(1) $\varphi$ is Hölder continuous of order $\theta$.

(2) If $0<s \leq r$ and $f \in \mathcal{F}$ then

$$
\int_{B(y, s)} f^{2} d \Gamma(\varphi, \varphi) \leq c_{1}(s / r)^{2 \theta}\left(\int_{B(y, 2 s)} d \Gamma(f, f)+\Psi(s)^{-1} \int_{B(y, 2 s)} f^{2} d m\right) .
$$


'CS' here refers to 'cutoff Sobolev'; this condition ensures the existence of a large class of cutoff functions with low energy. The main theorem of [BB3, BBK] is that $\mathrm{HK}(\Psi)$ is equivalent to $\mathrm{VD}+\mathrm{PI}(\Psi)+\mathrm{CS}(\Psi)$. While the condition $\mathrm{CS}(\Psi)$ is hard to verify, it is stable. Further, this stability allows estimates on (for example) the heat kernel on the Sierpinski carpet to be transferred to manifolds, graphs, or domains in $\mathbb{R}^{d}$ which are roughly isometric to the Sierpinski carpet. For rough isometries see [Kan], and a for more detailed discussion of this point see [BBK, Section 5].

We now introduce a simplication of the condition $\operatorname{CS}(\Psi)$, which controls the energy of cutoff functions in annuli.

Definition 1.9. Let $D_{0}, D_{1}$ be open subsets of $\mathcal{X}$ with $D_{0} \subset \bar{D}_{0} \subset D_{1}$, and let $U=$ $D_{1}-\bar{D}_{0}$. We say that condition $\operatorname{CSD}\left(D_{0}, D_{1}, \theta\right)$ holds if there exists a cutoff function $\varphi$ for $D_{0} \subset D_{1}$ such that if $f \in \mathcal{F}$ then,

$$
\int_{U} f^{2} d \Gamma(\varphi, \varphi) \leq \frac{1}{8} \int_{U} \varphi^{2} d \Gamma(f, f)+\theta \int_{U} f^{2} d m
$$

Definition 1.10 (Condition $(\mathrm{CSA}(\Psi))$. We say that condition $\mathrm{CSA}(\Psi)$ holds if there exists a constant $C_{S}$ such that for every $x \in \mathcal{X}, R>0, r>0$ the condition $\operatorname{CSD}\left(B(x, R), B(x, R+r), C_{S} \Psi(r)^{-1}\right)$ holds.

Remark 1.11. 1. If VD holds then CS $(\Psi)$ implies CSA $(\Psi)$ - see Lemma 5.7.

2. Note that $\operatorname{CSA}(\Psi)$ does not require the Hölder continuity of the cutoff function.

3. It is essential for the use of (1.17) in Lemma 3.3 that the constant in front of the first term on the right hand side is less than $\frac{1}{4}$. However, as we will see in Section 5, the inequality CSA $(\Psi)$ has a 'self-improving' property, which enables one to alter the weights of the two terms on the right-hand side.

4. It is easy to see by using linear cutoff functions that CSA(2) holds on any manifold.

5. The bound (1.17) is not symmetric between $\varphi$ and $1-\varphi$, but very often we will just use the fact that $\varphi \leq 1$ in the first term on the right hand side.

6. In view of Lemma 1.1 and the results of Section 5, the condition $\mathrm{CSA}(\Psi)$ is stable see Corollary 5.2 .

7. See [Bas] for the use of an inequality similar to $\mathrm{CS}(\Psi)$ to prove stability of the elliptic Harnack inequality for a class of graphs.

Our first main theorem is the following characterization of $\operatorname{UHK}(\Psi)$.

Theorem 1.12. Assume that $\mathcal{X}$ satisfies $V D$ and is unbounded in the metric $d$. The following are equivalent:

(1) $\mathrm{FK}(\Psi)$ and $\mathrm{CSA}(\Psi)$.

(2) $\operatorname{UHK}(\Psi)$.

Remark 1.13. (1) See [GH] for several other conditions equivalent to $\operatorname{UHK}(\beta)$. Note however that unlike (1) above, none of these were known to be stable under bounded perturbation of the Dirichlet form $\mathcal{E}$. 
(2) The reader may wonder if while $\mathrm{CSA}(\Psi)$ is sufficient for upper bounds, one needs the stronger $\mathrm{CS}(\Psi)$ to obtain lower bounds as well. However, we expect that $\mathrm{HK}(\Psi)$ is equivalent to $\mathrm{VD}+\mathrm{PI}(\Psi)+\mathrm{CSA}(\Psi)$. In fact, $\mathrm{VD}+\mathrm{PI}(\Psi)$ is enough to give $\mathrm{FK}(\Psi)$, so using Theorem 1.12 one obtains $\operatorname{UHK}(\Psi)$. Given this, the methods of Stroock and Saloff-Coste [SCS], and Fabes-Stroock [FS] should then lead to a matching lower bound.

The main theorem of [BB3, BBK] was proved using Moser's method [Mo1]. To prove the implication $(1) \Rightarrow(2)$ in Theorem 1.12 we first show in Proposition 2.3 that $\operatorname{CSA}(\Psi)$ gives a generalization of the 'Davies-Gaffney' bound of [D]. Next, we use techniques developed in Gro, CG to prove a mean value inequality for caloric functions (i.e. solutions of the heat equation), which leads to the pointwise bounds $\operatorname{UHK}(\Psi)$. For the easier implication $(2) \Rightarrow(1)$ we use the method of [BBK], but since $\operatorname{CSA}(\Psi)$ is rather simpler than $\mathrm{CS}(\Psi)$ the proof is much quicker.

Our second main result concerns stochastic completeness.

Definition 1.14. The process associated $X$ is called stochastically complete if $P_{t} 1=1$ $m$-a.e. for some (or equivalently all) $t>0$.

The energy measure $\Gamma$ defines in an intrinsic way a pseudo metric $\varrho$ on $(\mathcal{X}, m)$ by

$$
\varrho(x, y)=\sup \{f(y)-f(x): f \in \mathcal{F}, \quad d \Gamma(f, f) \leq d m\}
$$

called the intrinsic metric or Carathéodory metric. We will denote by $B_{\varrho}(x, r)=\{y \in$ $\mathcal{X}: \varrho(x, y)<r\}$ the open ball with center $x$ and radius $r$ w.r.t. the $\varrho$ metric. Further, we will use the notation

$$
\varrho(x, \infty):=\sup \left\{r>0: B_{\varrho}(x, r) \text { is relatively compact } \subset \mathcal{X}\right\} .
$$

If $\mathcal{X}$ is a Riemannian manifold and $\mathcal{E}(f, f)=\int|\nabla f|^{2} d \mu$, then $\varrho$ is just the Riemannian metric.

The pseudo-metric $\varrho$ is not always useful. For some fractal sets such as the Sierpinski carpet the measures $\Gamma(f, f)$ and $m$ are mutually singular - see [Hi]. In these cases the only functions $f$ satisfying the conditions of (1.18) are constants, and so $\varrho$ is identically zero.

The following theorem gives, in the manifold case, the best possible criterion for stochastic completeness in terms of volume growth.

Theorem 1.15 (Gr1, Gr2, St1]). Suppose that the metrics $\varrho$ and $d$ on $\mathcal{X}$ are equivalent, and all balls $B_{\varrho}(x, r)$ are relatively compact. We say that $(V G C)$ holds if for some $x \in \mathcal{X}$,

$$
\int_{1}^{\infty} \frac{r}{\log m\left(B_{\varrho}(x, r)\right)} d r=\infty
$$

If $(V G C)$ holds then $(\mathcal{X}, \mathcal{E})$ is stochastically complete.

Our second main theorem gives a criterion for stochastic completeness, in terms of a balance between the energy of cutoff functions between a sequence of compact sets, and the volume of the regions between these sets. 
Theorem 1.16. Let $D_{n}$ be an increasing sequence of open sets with compact closure, such that $\cup D_{n}=\mathcal{X}$. Write $U_{n}=D_{n+1}-D_{n}$. Let $\theta_{n}>0$ be such that $\operatorname{CSD}\left(D_{n}, D_{n+1}, \theta_{n}\right)$ holds for each $n$.

(a) Suppose that $\theta_{n} \leq c_{1}$ for all $n$. If

$$
\liminf _{n} \frac{\theta_{n} m\left(U_{n}\right)}{4^{n}}=0
$$

then stochastic completeness holds.

(b) Suppose $\theta_{n}=c_{0}^{2} n^{2}$, and there exists a constant $b>0$ such that

$$
m\left(U_{n}\right) \leq e^{2 b(\log n)^{2}} .
$$

Then stochastic completeness holds.

Remark 1.17. 1. Note that this Theorem does not involve the intrinsic metric $\varrho$.

2. We give an example below of a space $\mathcal{X}$ such that for some sufficiently large $R_{0}$ one has $\mathcal{X}=B_{\varrho}\left(x_{0}, R_{0}\right)$, but which is still stochastically complete.

3. In terms of volume growth, this Theorem gives a weaker criterion than the results of Gr1, St1. However since (1.21) only requires that a subsequence of annuli have small volume, there are manifolds for which Theorem 1.16 gives stochastic completeness, while the volume growth criterion of [Gr1, St1] fails.

4. The constant 4 in (1.20) is not best possible; it is related to the choice of $1 / 8$ in (1.17).

5. See Remark 6.1 for the case $\theta_{n} \asymp n^{2 \gamma}$ for $\gamma \in(0,1)$.

The layout of this paper is as follows. In Section 2 we show how CSA $(\Psi)$ can be used to give a generalization to the space-time scaling $\Psi$ of the 'Davies-Gaffney' bound obtained by Davies in [D]. In Section 3 we use CSA $(\Psi)$ to obtain a Cacciopoli type inequality. This is then used in Section 4 to obtain mean value inequalities, which lead to the upper heat kernel bound $\operatorname{UHK}(\Psi)$. In Section 5 we prove that $\operatorname{UHK}(\Psi)$ implies $\operatorname{CSA}(\Psi)$. Section 6 proves Theorem 1.16, and Section 7 gives examples, based on the 'pre-Sierpinski carpet', of spaces which are geodesically incomplete, or for which the criterion of [Gr1, St1] fails, but which are still stochastically complete.

We write $c, c^{\prime}$ to denote positive constants which may change on each appearance. Constants denoted $c_{i}$ will be the same through each argument. Constants related to fundamental properties of the space $\mathcal{X}$ or Dirichlet form, such as those in the volume doubling property, will be denoted $C$. and will be the same throughout each argument.

Acknowledgment. The authors wish to thank Rich Bass for several conversations on the topic of Remark 1.13(2). 


\section{Davies Gaffney estimate}

We begin by noting the following Cauchy-Schwarz inequality. Let $u, v \in \mathcal{F}, f, g \in$ $L^{\infty}(\mathcal{X}, m)$, and $\lambda>0$. Then

$$
\begin{aligned}
\int_{\mathcal{X}} f g d \Gamma(u, v) & =\int_{\mathcal{X}}\left(f / \lambda^{1 / 2}\right)\left(\lambda^{1 / 2} g\right) d \Gamma(u, v) \\
& \leq\left(\lambda^{-1} \int_{\mathcal{X}} f^{2} d \Gamma(u, u)\right)^{1 / 2} \cdot\left(\lambda \int_{\mathcal{X}} g^{2} d \Gamma(v, v)\right)^{1 / 2} \\
& \leq \frac{1}{2 \lambda} \int_{\mathcal{X}} f^{2} d \Gamma(u, u)+\frac{\lambda}{2} \int_{\mathcal{X}} g^{2} d \Gamma(v, v) .
\end{aligned}
$$

Let $D_{n}, n \geq 0$ be an increasing sequence of open subsets of $\mathcal{X}$ with $\bar{D}_{n} \subset D_{n+1}$. Suppose that $\operatorname{CSD}\left(D_{n}, D_{n+1}, \theta_{n}\right)$ holds for each $n$, and let $\varphi_{n}$ be the associated cutoff functions. Let $\left(a_{n}, n \geq 0\right)$ be an increasing sequence, with $a_{0} \geq 0$. Set

$$
\begin{aligned}
\varphi & =a_{0}+\sum_{n=0}^{\infty}\left(a_{n+1}-a_{n}\right)\left(1-\varphi_{n}\right), \\
b_{n} & =\frac{\left(a_{n+1}-a_{n}\right)}{a_{n}}, \quad b^{*}=\sup _{n} b_{n}, \\
C_{0} & =\sup _{n} b_{n}^{2} \theta_{n} .
\end{aligned}
$$

Lemma 2.1. Suppose $D_{n}, \varphi_{n}$ and $\varphi$ are as above. Then for any $u \in \mathcal{F}$

$$
\int_{\mathcal{X}} u^{2} d \Gamma(\varphi, \varphi) \leq \frac{\left(b^{*}\right)^{2}}{8} \int_{\mathcal{X}} \varphi^{2} d \Gamma(u, u)+C_{0} \int_{\mathcal{X}} \varphi^{2} u^{2} d m
$$

Proof. Let $U_{n}=D_{n+1}-D_{n}$, and note that $a_{n} \leq \varphi \leq a_{n+1}$ on $U_{n}$. Since $\Gamma\left(\varphi_{n}, \varphi_{m}\right)=0$ if $n \neq m$, using CSD,

$$
\begin{aligned}
\int u^{2} d \Gamma(\varphi, \varphi) & =\sum_{n}\left(a_{n+1}-a_{n}\right)^{2} \int_{U_{n}} u^{2} d \Gamma\left(\varphi_{n}, \varphi_{n}\right) \\
& \leq \sum_{n}\left(a_{n+1}-a_{n}\right)^{2}\left(\frac{1}{8} \int_{U_{n}} d \Gamma(u, u)+\theta_{n} \int_{U_{n}} u^{2} d m\right) \\
& \leq \sum_{n} b_{n}^{2}\left(\frac{1}{8} \int_{U_{n}} \varphi^{2} d \Gamma(u, u)+\theta_{n} \int_{U_{n}} \varphi^{2} u^{2} d m\right)
\end{aligned}
$$

proving (2.5).

We can use this to obtain an analogue of Lemma 1 of [D].

Proposition 2.2. Let $\varphi, a_{n}, b_{n}, \theta_{n}$ and $C_{0}$ be as above, and suppose that $b^{*} \leq 1$. Let $f$ have compact support. Set $u_{t}=P_{t} f$. Then

$$
\begin{aligned}
& \left\|u_{t} \varphi\right\|_{2} \leq\|f \varphi\|_{2} \exp \left(2 C_{0} t\right) \\
& \int_{0}^{t} \int \varphi^{2} d \Gamma\left(u_{s}, u_{s}\right) d s \leq 2\|f \varphi\|_{2}^{2} e^{4 C_{0} t}
\end{aligned}
$$


Proof. Given Lemma 2.1 the proof is as in [D]. Let $f$ have compact support and $u_{t}=P_{t} f$. Let $N \geq 1$, and set

$$
\begin{aligned}
\widetilde{\varphi}_{N} & =a_{0}+\sum_{n=0}^{N}\left(a_{n+1}-a_{n}\right)\left(1-\varphi_{n}\right), \\
h_{N}(t) & =\left\|u_{t} \widetilde{\varphi}_{N}\right\|_{2}^{2}=\int u_{t}^{2} \widetilde{\varphi}_{N}^{2} d m .
\end{aligned}
$$

Then since $u_{t} \in \mathcal{D}(\mathcal{L}), \widetilde{\varphi}_{N}^{2} u_{t} \in \mathcal{F}$,

$$
\begin{aligned}
h_{N}^{\prime}(t) & =2\left\langle\mathcal{L} u_{t}, \widetilde{\varphi}_{N}^{2} u_{t}\right\rangle=-2 \mathcal{E}\left(u_{t}, \widetilde{\varphi}_{N}^{2} u_{t}\right) \\
& =-2 \int_{\mathcal{X}} \widetilde{\varphi}_{N}^{2} d \Gamma\left(u_{t}, u_{t}\right)-4 \int_{\mathcal{X}} \widetilde{\varphi}_{N} u_{t} d \Gamma\left(u_{t}, \widetilde{\varphi}_{N}\right) .
\end{aligned}
$$

Using (2.1) with $\lambda=2$ to bound the second term,

$$
h_{N}^{\prime}(t) \leq-\int_{\mathcal{X}} \widetilde{\varphi}_{N}^{2} d \Gamma\left(u_{t}, u_{t}\right)+4 \int_{\mathcal{X}} u_{t}^{2} d \Gamma\left(\widetilde{\varphi}_{N}, \widetilde{\varphi}_{N}\right) .
$$

So by Lemma 2.1,

$$
\begin{aligned}
h_{N}^{\prime}(t) & \leq-\left(1-\frac{1}{2}\left(b^{*}\right)^{2}\right) \int_{\mathcal{X}} \widetilde{\varphi}_{N}^{2} d \Gamma\left(u_{t}, u_{t}\right)+4 C_{0} \int_{\mathcal{X}} \widetilde{\varphi}_{N}^{2} u_{t}^{2} d m \\
& \leq-\frac{1}{2} \int \widetilde{\varphi}_{N}^{2} d \Gamma\left(u_{t}, u_{t}\right)+4 C_{0} h_{N}(t) .
\end{aligned}
$$

Thus $h_{N}^{\prime} \leq 4 C_{0} h_{N}$, and hence $h_{N}(t) \leq h_{N}(0) \exp \left(4 C_{0} t\right)$. Integrating (2.10) we obtain

$$
h_{N}(t)-h_{N}(0)+\frac{1}{2} \int_{0}^{t} \int \widetilde{\varphi}_{N}^{2} d \Gamma\left(u_{s}, u_{s}\right) d s \leq\left\|f \widetilde{\varphi}_{N}\right\|_{2}^{2}\left(e^{4 C_{0} t}-1\right) .
$$

Letting $N \rightarrow \infty$ gives (2.7) and (2.6).

We can use this to obtain a generalization of the 'Davies-Gaffney' bound in [D].

Proposition 2.3. Suppose $\operatorname{CSA}(\Psi)$ holds. Let $x_{1}, x_{2} \in \mathcal{X}$ and let $d\left(x_{1}, x_{2}\right)=R$. If $f_{i} \in L^{2}$ have support in $A_{i}=B\left(x_{i}, R / 4\right)$ then

$$
\left\langle P_{t} f_{1}, f_{2}\right\rangle \leq c_{1}|| f_{1}\left\|_{2}\right\| f_{2} \|_{2} \exp \left(-c_{2} \Phi(R, t)\right) .
$$

Here $c_{i}$ depend only on $C_{S}, \beta$ and $\beta_{L}$.

Proof. First note that

$$
\left\langle P_{t} f_{1}, f_{2}\right\rangle \leq\left\|P_{t} f_{1}\right\|_{2}\left\|f_{2}\right\|_{2} \leq\left\|f_{1}\right\|_{2}\left\|f_{2}\right\|_{2} ;
$$

adjusting the constants $c_{i}$ this is enough to give (2.11) if $\Phi(R, t)$ is small. Next, it is enough to prove (2.11) when $\left\|f_{i}\right\|_{2}=1$, so we assume this. 
Choose $m \geq 1$, let $r=R / 2 m$, and $D_{k}=B\left(x_{1}, \frac{1}{4} R+k r\right), k \geq 0$. Let $\varphi_{k}$ be cutoff functions for $D_{k} \subset D_{k+1}$, for $k \geq 0$. Set $a_{k}=2^{k \wedge m}$, and define $\varphi$ as in (2.2). Note that $b^{*}=(2-1)^{2}=1, \theta_{k}=C_{S} \Psi(r)^{-1}$, and so $C_{0}=C_{S} \Psi(r)^{-1}$.

Then writing $u_{t}=P_{t} f_{1}$, as in [D, Theorem 2] we have

$$
\begin{aligned}
\left\langle P_{t} f_{1}, f_{2}\right\rangle & =\left\langle\varphi P_{t} f_{1}, \varphi^{-1} f_{2}\right\rangle \\
& \leq\left\|\varphi u_{t}\right\|_{2}\left\|\varphi^{-1} f_{2}\right\|_{2} \\
& \leq\left\|\varphi f_{1}\right\|_{2} \exp \left(2 C_{S} \Psi(r)^{-1} t\right)\left\|\varphi^{-1} f_{2}\right\|_{2} \\
& \leq \exp \left(2 C_{S} \Psi(r)^{-1} t\right)\left(\sup _{A_{1}} \varphi\right)\left(\sup _{A_{2}} \varphi^{-1}\right) .
\end{aligned}
$$

The construction of $\varphi$ gives $\varphi=1$ on $A_{1}$, and $\varphi=2^{m}$ on $A_{2}$. So

$$
\log \left\langle P_{t} f_{1}, f_{2}\right\rangle \leq-\left(m \log 2-\frac{2 C_{S} t}{\Psi(R / m)}\right)=-\log 2\left(m-\frac{c_{3} t}{\Psi(R / m)}\right) .
$$

It remains to choose $m \in \mathbb{N}$ so as to obtain the bound (2.11), and we need to consider several cases.

Case 1. $t \geq \Psi(R)$. By Lemma 1.5 we have $\Phi(R, t) \asymp 1$, and adjusting the constant $c_{1}$ we obtain (2.11) from (2.12).

Case 2. $R \leq t \leq \Psi(R)$. Then $R \geq 1$, so we have $R \leq t<R^{\beta}$. We will choose $m \leq R$, so the final term in (2.14) is

$$
m\left(1-\frac{c_{3} t m^{\beta-1}}{R^{\beta}}\right) .
$$

We wish to choose $m$ so that $c_{3} t m^{\beta-1} R^{-\beta} \in[1 / 3,2 / 3]$, and this will be possible provided $R^{\beta} / t$ is greater than some constant $c_{4}$ (depending only on $c_{3}$ and $\beta$ ). We then have $m \asymp\left(R^{\beta} / t\right)^{1 /(\beta-1)}$, and hence we obtain the bound (2.11). If $R^{\beta} / t<c_{4}$ then $\Phi(R, t) \leq c_{5}$ and again we obtain (2.11) from (2.12).

Case 3. $t<R$ and $t \leq \Psi(R)$. In this case we will choose $m>R$, so that $\Psi(R / m)=$ $(R / m)^{\beta_{L}}$. If $R \leq 1$ then $\Psi(R)=R^{\beta_{L}}$ and so the argument is as in Case 2. If $R>1$ then $t<R<R^{\beta_{L}}$, so again we can proceed as in Case 2 .

\section{Cacciopoli and mean value inequalities}

In this section we prove a mean value inequality as in Gr0, Section 3]. We begin by seeing that $\operatorname{CSA}(\Psi)$ enables us to prove a Cacciopoli inequality similar to Gr0, Lemma 3.1]. To that aim we need to give a definition of caloric functions in the general context of metric measure spaces.

Definition 3.1. Let $I$ be an interval in $\mathbb{R}$. We say that a function $u: I \rightarrow L^{2}(\mathcal{X}, m)$ is weakly differentiable at $t_{0} \in I$ if for any $f \in L^{2}(\mathcal{X}, m)$ the function $\langle u(t), f\rangle$ is differentiable at $t_{0}$. By the principle of uniform boundedness, in this case there is a function $w \in L^{2}(\mathcal{X}, m)$ such that

$$
\lim _{t \rightarrow t_{0}}\left(\frac{u(t)-u\left(t_{0}\right)}{t-t_{0}}, f\right)=\langle w, f\rangle
$$


for all $f \in L^{2}(\mathcal{X}, m)$. We refer to the function $w$ as the weak derivative of the function $u$ at $t_{0}$ and write $w=\frac{\partial}{\partial t} u\left(t_{0}\right)=u_{t}\left(t_{0}\right)$.

Definition 3.2. Consider a function $u: I \rightarrow \mathcal{F}$ and let $\Omega$ be an open subset of $\mathcal{X}$. We say that $u$ is a caloric function in $I \times \Omega$ if $u$ is weakly differentiable in the space $L^{2}(\Omega)$ at any $t \in I$ and, for any non-negative $f \in \mathcal{F}_{\Omega}$ and for any $t \in I$,

$$
\left\langle u_{t}, f\right\rangle+\mathcal{E}(u, f)=0 .
$$

Lemma 3.3. (Cacciopoli inequality.) Let $x_{0} \in \mathcal{X}, B=B\left(x_{0}, R\right), r<R$ and $B^{\prime}=$ $B\left(x_{0}, R-r\right)$. Suppose that $\operatorname{CSD}\left(B^{\prime}, B, \theta\right)$ holds, and let $\varphi$ be the associated cutoff function for $B^{\prime} \subset B$. Let $T>0$, and set $Q=B \times(0, T)$. Let $k(t)$ be a Lipschitz function of $t$ with $k(0)=0,0 \leq k \leq 1$ and $\left\|k^{\prime}\right\|_{\infty}=K$. Let $u=u(x, t)$ be a non-negative caloric function, and $v=(u-\theta)_{+}$, where $\theta>0$. Set

$$
\eta(x, t)=\varphi(x) k(t) .
$$

Then

$$
\int_{B} v(x, T)^{2} \eta(x, T)^{2} m(d x)+\frac{2}{9} \int_{Q} d \Gamma(\eta v, \eta v) d t \leq 2\left(\frac{20}{9} \theta+K\right) \int_{Q} v^{2} d m d t .
$$

Proof. Since $k(0)=0$ we have, writing $v_{t}=\partial v / \partial t$,

$$
\frac{1}{2} \int_{B} v(x, T)^{2} \eta(x, T)^{2} m(d x)=\int_{Q} v v_{t} \eta^{2} d m d t+\int_{Q} v^{2} \eta \eta_{t} d m d t .
$$

Using the fact that $u$ is caloric we get

$$
\begin{aligned}
\int_{Q} \eta^{2} v v_{t} d m d t & =\int_{Q} \mathbb{1}_{\{u>\theta\}} \eta^{2} v u_{t} d m d t=-\int_{Q} d \Gamma\left(\eta^{2} v, u\right) \\
& =-\int_{Q} \eta^{2} d \Gamma(v, v) d t-2 \int_{Q} v \eta d \Gamma(v, \eta) d t .
\end{aligned}
$$

Further,

$$
\int_{B} d \Gamma(v \varphi, v \varphi) \leq 2 \int_{B} \varphi^{2} d \Gamma(v, v)+2 \int_{B} v^{2} d \Gamma(\varphi, \varphi) .
$$

Let $\lambda>0$. Then using (2.1)

$$
-\int_{B} \varphi^{2} d \Gamma(v, v)-2 \int_{B} v \varphi d \Gamma(v, \varphi) \leq\left(-1+\lambda^{-1}\right) \int_{B} \varphi^{2} d \Gamma(v, v)+\lambda \int_{B} v^{2} d \Gamma(\varphi, \varphi) .
$$

Taking $\lambda=2$ and using (3.4) and CSD we obtain

$$
\begin{aligned}
-\int_{B} \varphi^{2} d \Gamma(v, v)-2 & \int_{B} v \varphi d \Gamma(v, \varphi)+a \int_{B} d \Gamma(v \varphi, v \varphi) \\
& \leq\left(-\frac{1}{2}+2 a\right) \int_{B} \varphi^{2} d \Gamma(v, v)+(2+2 a) \int_{B} v^{2} d \Gamma(\varphi, \varphi) \\
& \leq\left(-\frac{1}{2}+2 a+(2+2 a) \frac{1}{8}\right) \int_{B} \varphi^{2} d \Gamma(v, v)+(2+2 a) \theta \int_{B} v^{2} d m \\
& =\frac{20}{9} \theta \int_{B} v^{2} d m
\end{aligned}
$$


if $a=1 / 9$. Multiplying this inequality by $k(t)^{2}$ and integrating gives

$$
\begin{aligned}
-\int_{Q} \eta^{2} d \Gamma(v, v) d t & -2 \int_{Q} v \eta d \Gamma(v, \eta) d t+\frac{1}{9} \int_{Q} d \Gamma(v \eta, v \eta) d t \\
& \leq \frac{20}{9} \theta \int_{0}^{T} \int_{B} v^{2} k(t)^{2} d m d t \leq \frac{20}{9} \theta \int_{Q} v^{2} d m d t .
\end{aligned}
$$

Combining this with (3.2) and (3.3) we obtain

$$
\begin{aligned}
\int_{B} v(x, T)^{2} \eta(x, T)^{2} m(d x)+\frac{2}{9} & \int_{Q} d \Gamma(v \eta, v \eta) d t \\
& \leq 2 \int_{Q} v^{2} \eta \eta_{t} d m d t+\frac{40}{9} \theta \int_{Q} v^{2} d m d t \\
& \leq\left(2 K+\frac{40}{9} \theta\right) \int_{Q} v^{2} d m d t .
\end{aligned}
$$

In the final line we used the fact that $\eta \leq 1$.

Remark 3.4. Note that to obtain (3.5) we needed that the constant in the first term on the right of (1.17) was less than $1 / 4$.

The key step in the proof of the mean value inequality is the following comparison over cylinders. For a cylinder $Q \subset \mathcal{X} \times \mathbb{R}_{+}$and a function $w$ write

$$
I(w, Q)=\int_{Q} w^{2} d m d t .
$$

Lemma 3.5. (See [Gr0, Lemma 3.2].) Suppose $\mathrm{FK}(\Psi)$ and $\operatorname{CSA}(\Psi)$ hold. Let $\widetilde{u}$ be a caloric function in $Q=B\left(x_{0}, R\right) \times(0, T)$. Let $u=\widetilde{u}_{+}, \theta>0$ and $v=(u-\theta)_{+}$. Let $0<T_{1}<T, R_{1} \in\left(\frac{1}{2} R, R\right), Q_{1}=B\left(x_{0}, R_{1}\right) \times\left(T_{1}, T\right)$,

$$
I=I(u, Q), \quad I_{1}=I\left(v, Q_{1}\right),
$$

and $\delta=T_{1} \wedge \Psi\left(R-R_{1}\right)$. Then

$$
I_{1} \leq \frac{c_{1} I^{1+\nu} \Psi(R)}{\delta^{1+\nu} \theta^{2 \nu} m(B)^{\nu}} .
$$

Proof. Let $B=B\left(x_{0}, R\right), B^{\prime}=B\left(x_{0}, \frac{1}{2}\left(R+R_{1}\right)\right)$ and $B_{1}=B\left(x_{0}, R_{1}\right)$. Set

$$
D_{t}=\left\{x \in B^{\prime}: u(x, t)>\theta\right\} .
$$

Let $\varphi$ be a cutoff function for $B_{1} \subset B^{\prime}, k(t)=1 \wedge\left(t / T_{1}\right)$, and $\eta(x, t)=\varphi(x) k(t)$. 
As in [Gr0] the proof uses five inequalities:

$$
\begin{aligned}
\int_{B^{\prime}} u\left(x, t_{0}\right)^{2} d m & \leq c_{0} \delta^{-1} \int_{Q} u^{2} d m d t \text { for } t_{0} \in\left(T_{1}, T\right), \\
\int_{Q} d \Gamma(v \eta, v \eta) d t & \leq c_{0} \delta^{-1} \int_{Q} u^{2} d m d t \\
\int_{B} d \Gamma(v \eta, v \eta) & \geq \lambda_{1}\left(D_{t}\right) \int_{B} v^{2} \eta^{2} d m \text { for } t \in(0, T), \\
\lambda_{1}\left(D_{t}\right) & \geq C_{F} m(B)^{\nu} \Psi(R)^{-1} m\left(D_{t}\right)^{-\nu} \\
m\left(D_{t}\right) & \leq \theta^{-2} \int_{B^{\prime}} u(x, t)^{2} d m .
\end{aligned}
$$

Of these, (3.9) is immediate from the variational definition of $\lambda_{1}$, (3.10) is the Faber-Krahn inequality (1.8), and (3.11) is just Markov's inequality. So it remains to prove (3.7) and (3.8).

The inequality (3.8) is immediate from (3.1). Since $\left\|k^{\prime}\right\|_{\infty}=1 / T_{1}$ we have the constant on the right side of (3.1) is $c\left(\Psi\left(R-R_{1}\right)^{-1}+T_{1}^{-1}\right) \leq c^{\prime} \delta^{-1}$. So

$$
\frac{2}{9} \int_{Q} d \Gamma(v \eta, v \eta) d t \leq c \delta^{-1} \int_{Q} u^{2} d m d t .
$$

For (3.7) let $\widetilde{\varphi}$ be a cutoff function for $B^{\prime} \subset B$ and $\widetilde{\eta}(x, t)=\widetilde{\varphi}(x) k(t)$. Then by (3.1) applied to $u$ in the cylinder $Q_{t}=B \times(0, t)$,

$$
\int_{B} u(x, t)^{2} \widetilde{\eta}(x, t)^{2} m(d x) \leq c \delta^{-1} \int_{Q_{t}} u^{2} d m d t \leq c \delta^{-1} \int_{Q} u^{2} d m d t .
$$

The rest of the argument is as in Gr0].

\section{$4 \quad$ Heat kernel upper bounds}

These bounds can now be proved by the methods of [CG], which in turn uses ideas in Gr0]. Since [CG] is written in the graph context, and both of these papers just consider the case $\Psi(r)=r^{2}$, we give details. In particular we need to be more careful in our handling of exceptional sets; issues with these do not arise for the manifolds or graphs treated in [Gr0, CG]. Note that VD implies that there exists a constant $\alpha<\infty$ such that

$$
\frac{V(x, R)}{V(y, r)} \leq C_{G}\left(\frac{d(x, y)+R}{r}\right)^{\alpha}, \quad 0<r<R, x, y \in \mathcal{X}
$$

Define the measure $\widetilde{m}(d x, d s)=m(d x) d s$ on $\mathcal{X} \times \mathbb{R}$. Given a cylinder $Q \subset \mathcal{X} \times \mathbb{R}$ and $u: Q \rightarrow \mathbb{R}$ we write ess $\sup u$ for the essential supremum with respect to the measure $\widetilde{m}$.

Define

$$
\beta_{2}=\beta_{L} \vee \beta, \quad \beta_{1}=\beta_{L} \wedge \beta
$$


and note that if $0<r<R$,

$$
\left(\frac{R}{r}\right)^{\beta_{1}} \leq \frac{\Psi(R)}{\Psi(r)} \leq\left(\frac{R}{r}\right)^{\beta_{2}}, \quad\left(\frac{R}{r}\right)^{1 / \beta_{2}} \leq \frac{\Psi^{-1}(R)}{\Psi^{-1}(r)} \leq\left(\frac{R}{r}\right)^{1 / \beta_{1}}
$$

Write

$$
F(R, T)=\frac{T}{\Psi(R)} \vee\left(\frac{\Psi(R)}{T}\right)^{\alpha / \beta_{1}}
$$

Proposition 4.1. ( $L^{2}$ Mean value inequality). Set $Q=B\left(x_{0}, R\right) \times(0, T)$. Assume $\operatorname{CSA}(\Psi)$ and $\mathrm{FK}(\Psi)$ hold, and let $u \geq 0$ be caloric in $Q$. Then if $Q_{\infty}=B\left(x_{0}, R / 2\right) \times$ $(T / 2, T)$,

$$
\operatorname{ess}_{\sup } u(x, s)^{2} \leq \frac{c_{0} F(R, T)}{T V\left(x_{0}, R\right)} \int_{Q} u(x, s)^{2} \widetilde{m}(d x, d s) .
$$

Proof. (See the proof of Gr0, Theorem 3.1].) It is sufficient to consider the case $T=$ $\Psi(R)$. Indeed, suppose (4.5) holds in this case, and let $T=\lambda \Psi(R)$.

If $\lambda \in(0,1)$ let $r$ be such that $\Psi(r)=T$. We can cover $B^{\prime}=B\left(x_{0}, R / 2\right)$ by balls $B\left(z_{i}, r / 2\right)$ such that each $B\left(z_{i}, r\right) \subset B\left(x_{0}, R\right)$. Let $Q_{i}=B\left(z_{i}, r\right) \times(0, T)$, and $Q_{i, \infty}=$ $B\left(z_{i}, r / 2\right) \times(T / 2, T)$. Note that by (4.3)

$$
\frac{V\left(x_{0}, R\right)}{V\left(x_{0}, r\right)} \leq C_{G}\left(\frac{R}{r}\right)^{\alpha}=C_{G}\left(\frac{\Psi^{-1}(T / \lambda)}{\Psi^{-1}(T)}\right)^{\alpha} \leq C_{G} \lambda^{-\alpha / \beta_{1}}
$$

Then

$$
\operatorname{ess} \sup _{Q_{\infty}} u(x, s)^{2} \leq \max _{i} \operatorname{ess} \sup _{Q_{i, \infty}} u(x, s)^{2},
$$

and for each $i$, using (4.6),

$$
\begin{aligned}
\operatorname{ess} \sup _{Q_{i, \infty}} u(x, s)^{2} & \leq \frac{c_{0}}{T V\left(x_{0}, r\right)} \int_{Q_{i}} u^{2} d \widetilde{m} \\
& \leq \frac{c_{0}}{T V\left(x_{0}, R\right)} \frac{V\left(x_{0}, R\right)}{V\left(x_{0}, r\right)} \int_{Q} u^{2} d \widetilde{m} \\
& \leq \frac{c_{0} C_{G} \lambda^{-\alpha / \beta_{1}}}{T V\left(x_{0}, R\right)} \int_{Q} u^{2} d \widetilde{m} .
\end{aligned}
$$

Similarly if $T=\lambda \Psi(R)$ with $\lambda>1$ then applying (4.5) to a sequence of cylinders $\left(T_{i}-\right.$ $\left.\Psi(R), T_{i}\right) \times B\left(x_{0}, R\right)$ one obtains

$$
\operatorname{ess} \sup _{Q_{\infty}} u(x, s)^{2} \leq \frac{c_{0} \lambda}{T V\left(x_{0}, R\right)} \int_{Q} u(x, s)^{2} d \widetilde{m} .
$$

Now let $T=\Psi(R)$. Let $\delta_{k}, k=0,1, \ldots$ be a sequence to be chosen later, and let $\left(r_{k}\right)$ and $\left(t_{k}\right)$ be sequences such that $r_{0}=R, t_{0}=0$,

$$
\delta_{k+1}=\Psi\left(r_{k}-r_{k+1}\right)=t_{k+1}-t_{k},
$$


and

$$
R=r_{0}>r_{1}>\cdots>r_{k}>\cdots>R / 2, \quad 0=t_{0}<t_{1}<\cdots<t_{k}<\cdots<T / 2 .
$$

Let $Q_{k}=B\left(x_{0}, r_{k}\right) \times\left(t_{k}, T\right)$. Let $\theta>0$ (also to be chosen later) and set $\alpha_{k}=\left(1-2^{-k}\right) \theta$, $u_{k}=\left(u-\alpha_{k}\right)_{+}$, and

$$
I_{k}=\int_{Q_{k}} u_{k}^{2} d \widetilde{m}
$$

Let $\theta_{k}=\alpha_{k+1}-\alpha_{k}$. Then by (3.6) applied to the function $u_{k}$ in $Q_{k+1} \subset Q_{k}$,

$$
I_{k+1} \leq I_{k} \frac{c_{1} I_{k}^{\nu} \Psi\left(r_{k}\right)}{\delta_{k+1}^{1+\nu} \theta_{k}^{2 \nu} m\left(B\left(x_{0}, r_{k}\right)\right)^{\nu}} \leq I_{k} \frac{c_{2} \Psi(R) 2^{2 \nu(k+1)} I_{k}^{\nu}}{V\left(x_{0}, R\right)^{\nu} \theta^{2 \nu} \delta_{k+1}^{1+\nu}}
$$

Let $A=c_{2} \Psi(R) 4^{\nu} / V\left(x_{0}, R\right)^{\nu} \theta^{2 \nu}$. Now choose $M \geq 2$ and $b>4$ such that $M^{1 / \beta_{2}} \geq 2$, and $(4 / b)^{1 /(1+\nu)}=M^{-1}$. Choose $\delta_{k+1}$ such that

$$
\frac{A 2^{2 \nu k} I_{k}^{\nu}}{\delta_{k+1}^{1+\nu}}=b^{-1}, \quad k=0,1, \ldots
$$

With this choice of $\left(\delta_{k}\right)$ we have $I_{k} \leq b^{-k} I_{0}$, and consequently

$$
\delta_{k+1}=\left(A b 4^{k \nu} I_{k}^{\nu}\right)^{1 /(1+\nu)} \leq\left(A b I_{0}^{\nu}\right)^{1 /(1+\nu)}(4 / b)^{k \nu /(1+\nu)}=A_{1} M^{-k},
$$

where $A_{1}=\left(A b I_{0}^{\nu}\right)^{1 /(1+\nu)}$. In order that the condition (4.8) should hold, we need

$$
\begin{aligned}
& \sum_{k=1}^{\infty} \delta_{k} \leq T / 2, \\
& \sum_{k=1}^{\infty} \Psi^{-1}\left(\delta_{k}\right) \leq R / 2 .
\end{aligned}
$$

We have

$$
\sum_{k=1}^{\infty} \Psi^{-1}\left(\delta_{k}\right) \leq \sum_{k=1}^{\infty} \Psi^{-1}\left(A_{1} M^{-k}\right) \leq c_{3} \Psi^{-1}\left(A_{1}\right) \sum_{k=1}^{\infty} M^{-k / \beta_{2}} \leq 2 c_{3} \Psi^{-1}\left(A_{1}\right),
$$

and

$$
\sum_{k=1}^{\infty} \delta_{k} \leq 2 A_{1}
$$

So for (4.8) to hold it is enough that

$$
A_{1}=\left(\frac{c_{2} 4^{\nu} b I_{0}^{\nu} \Psi(R)}{V\left(x_{0}, R\right)^{\nu} \theta^{2 \nu}}\right)^{1 /(1+\nu)} \leq(T / 4) \wedge \Psi\left(R / 4 c_{3}\right) .
$$

For this it is enough if $\theta$ is chosen large enough so that

$$
\frac{I_{0}^{\nu} \Psi(R)}{V\left(x_{0}, R\right)^{\nu} \theta^{2 \nu}} \leq c_{4} \Psi(R)^{1+\nu}
$$


and so we can take

$$
\theta^{2}=\frac{c_{5} I_{0}}{\Psi(R) V\left(x_{0}, R\right)} .
$$

We then have $I_{k} \rightarrow 0$ as $k \rightarrow \infty$, and hence

$$
\int_{Q_{\infty}}(u(x, s)-\theta)_{+}^{2} \widetilde{m}(d x, d s) \leq \inf _{k} I_{k}=0,
$$

which implies that $u(x, s) \leq \theta \widetilde{m}$ a.e. on $Q_{\infty}$.

We now give an $L^{1}$ mean value inequality.

Proposition 4.2. ( $L^{1}$ Mean value inequality). Assume $\operatorname{FK}(\Psi)$ and $\operatorname{CSA}(\Psi)$ hold. Let $R>0, T=\Psi(R)$, let $Q=B\left(x_{0}, R\right) \times(0, T)$, and let $u \geq 0$ be caloric in $Q$. Then writing $Q^{\prime}=B\left(x_{0}, 2 R / 3\right) \times(T / 2, T)$,

$$
\operatorname{ess} \sup _{Q^{\prime}} u(x, s) \leq \frac{c_{1} F(R, T)}{T V\left(x_{0}, R\right)} \int_{Q} u(x, s) \widetilde{m}(d x, d s) .
$$

Proof. This follows from the $L^{2}$ mean value inequality by quite general arguments, which use only VD - see p. 688-691 of [CG]. As with Proposition 4.1, it is enough to consider the case $T=\Psi(R)$.

In order to obtain heat kernel bounds from the mean value theorem, we need better control of the exceptional set. We will use regularity results from [GT], and to use these we need to consider the killed heat kernel. For $D \subset \mathcal{X}$ write $\left(P_{t}^{D}\right)$ for the semigroup of $X$ killed on exiting $D$. Then if $\mathrm{FK}(\Psi)$ holds, by [GH, Lemma 5.5] the semigroup $\left(P_{t}^{D}\right)$ is ultracontractive, that is there exists a left continuous function $\gamma(t)$ such that

$$
\left\|P_{t}^{D} f\right\|_{\infty} \leq \gamma(t)\|f\|_{1}, \quad t>0, f \in L^{1}(\mathcal{X}) \cap L^{2}(\mathcal{X}) .
$$

(In fact we have $\gamma(t)=c(a t)^{-1 / \nu}$ with $a=a(n)=m\left(D_{n}\right)^{\nu} / \Psi(n R)$ ). Consequently we will be able to use [GT, Theorem 2.12] to obtain estimates which hold on $\mathcal{X}-\mathcal{N}$, where $\mathcal{N}$ is a properly exceptional set.

Lemma 4.3. Assume $\mathrm{FK}(\Psi)$ and $\operatorname{CSA}(\Psi)$. Let $x_{0}, x_{1} \in \mathcal{X}_{0}, T>0,0<t \leq T$, and $r=\Psi(t)$. Let $R_{0}>R$ and $D=B\left(x_{0}, R_{0}\right)$. Then for m-a.a. $y \in B\left(x_{0}, r / 2\right)$,

$$
p_{T}^{D}\left(x_{1}, y\right) \leq \frac{c_{1}}{t V\left(x_{0}, r\right)} \int_{T-t / 2}^{T+t / 2} \int_{B(x, r)} p_{s}^{D}\left(x_{1}, y^{\prime}\right) \widetilde{m}\left(d y^{\prime}, d s\right) .
$$

Proof. Set $Q=B\left(x_{0}, r\right) \times(T-t / 2, T+t / 2)$, and $Q^{\prime}=B\left(x_{0}, r / 2\right) \times(T, T+t / 2)$. Since $u(y, s)=p_{s}^{D}\left(x_{1}, y\right)$ is caloric in $Q$, by Proposition 4.2

$$
\operatorname{ess} \sup _{(y, s) \in Q^{\prime}} p_{s}^{D}\left(x_{1}, y\right) \leq \frac{c_{1}}{t V\left(x_{0}, r\right)} \int_{Q} p_{s}^{D}\left(x_{1}, y^{\prime}\right) \widetilde{m}\left(d y^{\prime}, d s\right) .
$$


Setting

$$
A=\frac{c_{1}}{t V\left(x_{0}, r\right)} \int_{Q} p_{s}^{D}\left(x_{1}, y^{\prime}\right) \widetilde{m}\left(d y^{\prime}, d s\right),
$$

the right side of (4.18) is bounded by $A$.

Thus there exists a subset $I \subset(T, T+t / 2)$ of full measure such that if $T^{\prime} \in I$ then

$$
p_{T^{\prime}}^{D}\left(x_{1}, y\right) \leq A, \quad \text { for } m \text {-a.a. } y \in B\left(x_{0}, r / 2\right) \text {. }
$$

Write $g(y)=p_{T}^{D}\left(x_{1}, y\right)$. Then the $L^{2}$-continuity of $\left(P_{t}^{D}\right)$ implies that $P_{h}^{D} g \rightarrow g m$-a.e. as $h \rightarrow 0$. Taking the limit along a sequence $h_{k}$ such that $T+h_{k} \in I$ for each $k$, it follows that $p_{T}^{D}\left(x_{1}, y\right) \leq A$ for $m$-a.a. $y \in B\left(x_{0}, r / 2\right)$.

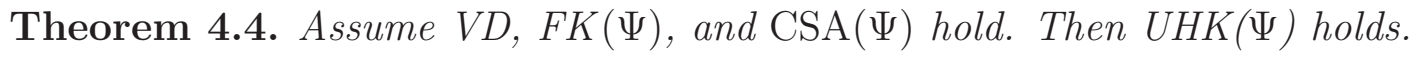

Proof. We use the argument of [CG], but need extra care because of exceptional sets. Fix $x_{0}, y_{0} \in \mathcal{X}_{0}, T>0$, and let $R=d(x, y)$. Let $R_{0}>4 R$, and let $D=B\left(x_{0}, R_{0}\right)$. Set $T_{0}=T / 2$ and $r=\Psi^{-1}\left(T_{0}\right)$. Let $Q(z)=B(z, r) \times\left(T-T_{0} / 2, T+T_{0} / 2\right)$. Let $r^{\prime}<R / 4 \wedge r / 2$, and $g_{1}$ and $g_{2}$ be non-negative bounded functions with supports in $B\left(x_{0}, r^{\prime}\right)$ and $B\left(y_{0}, r^{\prime}\right)$ respectively, such that $\int g_{1}=\int g_{2}=1$. Set

$$
I=\iint p_{T}^{D}(x, y) g_{1}(x) g_{2}(y) m(d x) m(d y) .
$$

Let $x \in B\left(x_{0}, R / 4\right)$. Then applying (4.17) to the caloric function $u(y, s)=p_{s}^{D}(x, y)$ in $Q\left(y_{0}\right)$, we have

$$
p_{T}^{D}(x, y) \leq \frac{c_{1}}{T_{0} V\left(y_{0}, r\right)} \int_{T-T_{0} / 2}^{T+T_{0} / 2} \int_{B\left(y_{0}, r\right)} p_{s}^{D}\left(x, y^{\prime}\right) \widetilde{m}\left(d y^{\prime}, d s\right)
$$

for $m$-a.a. $y \in B\left(y_{0}, r / 2\right)$. Hence

$$
\begin{aligned}
I & \leq \frac{c_{1}}{T_{0} V\left(y_{0}, r\right)} \iint g_{1}(x) g_{2}(y) m(d x) m(d y) \int_{Q\left(y_{0}\right)} p_{s}^{D}\left(x, y^{\prime}\right) \widetilde{m}\left(d y^{\prime}, d s\right) \\
& =\frac{c_{1}}{T_{0} V\left(y_{0}, r\right)} \int_{B\left(y_{0}, r\right)} \int_{T-T_{0} / 2}^{T+T_{0} / 2} \int g_{1}(x) p_{s}^{D}\left(x, y^{\prime}\right) \widetilde{m}\left(d y^{\prime}, d s\right) m(d x) .
\end{aligned}
$$

If $y^{\prime} \in B\left(y_{0}, r\right)$ and $s \in\left(T-T_{0} / 2, T+T_{0} / 2\right)$, then by considering the cylinder $B\left(x_{0}, r\right) \times$ $\left(s-T_{0} / 2, s+T_{0} / 2\right)$, we have by (4.17), for $m$-a.a. $x \in B\left(x_{0}, r / 2\right)$,

$$
\begin{aligned}
p_{s}^{D}\left(y^{\prime}, x\right) & \leq \frac{c_{1}}{T_{0} V\left(x_{0}, r\right)} \int_{s-T_{0} / 2}^{s+T_{0} / 2} \int_{B\left(x_{0}, r\right)} p_{s^{\prime}}^{D}\left(y^{\prime}, x^{\prime}\right) \widetilde{m}\left(d x^{\prime}, d s^{\prime}\right) \\
& \leq \frac{c_{1}}{T_{0} V\left(x_{0}, r\right)} \int_{T-T_{0}}^{T+T_{0}} \int_{B\left(x_{0}, r\right)} p_{s^{\prime}}^{D}\left(x^{\prime}, y^{\prime}\right) \widetilde{m}\left(d x^{\prime}, d s^{\prime}\right)
\end{aligned}
$$


Substituting this into the final term in (4.21), we obtain

$$
\begin{aligned}
I \leq & \frac{c_{1}^{2}}{T_{0}^{2} V\left(y_{0}, r\right) V\left(x_{0}, r\right)} \int_{B\left(y_{0}, r\right)} m\left(d y^{\prime}\right) \int_{T-T_{0} / 2}^{T+T_{0} / 2} d s \\
& \times \int m(d x) g_{1}(x) \int_{T-2 T_{0}}^{T+2 T_{0}} d s^{\prime} \int_{B\left(x_{0}, r\right)} m\left(d x^{\prime}\right) p_{s^{\prime}}^{D}\left(x^{\prime}, y^{\prime}\right) \\
& =\frac{c_{1}^{2}}{T_{0} V\left(y_{0}, r\right) V\left(x_{0}, r\right)} \int_{B\left(y_{0}, r\right)} m\left(d y^{\prime}\right) \int_{B\left(x_{0}, r\right)} m\left(d x^{\prime}\right) \int_{T-T_{0}}^{T+T_{0}} d s^{\prime} p_{s^{\prime}}^{D}\left(x^{\prime}, y^{\prime}\right) \\
& \leq \frac{2 c_{1}^{2}}{T V\left(y_{0}, r\right) V\left(x_{0}, r\right)} \int_{T / 2}^{3 T / 2}\left\langle P_{s} f_{x}, f_{y}\right\rangle d s,
\end{aligned}
$$

where $f_{x}=1_{B\left(x_{0}, r\right)}$ and $f_{y}=1_{B\left(y_{0}, r\right)}$.

If $r<R / 4$ then the Davies Gaffney bound Proposition 2.3 implies that for $T / 2 \leq s \leq$ $2 T$

$$
\begin{aligned}
\left\langle P_{s} f_{x}, f_{y}\right\rangle & \leq c_{2} V\left(x_{0}, r\right)^{1 / 2} V\left(y_{0}, r\right)^{1 / 2} \exp \left(-c_{2} \Phi(R, s)\right) \\
& \leq c_{2} V\left(x_{0}, r\right)^{1 / 2} V\left(y_{0}, r\right)^{1 / 2} \exp \left(-c_{3} \Phi(R, T)\right)
\end{aligned}
$$

If $r \geq R / 4$ we still have by (2.12)

$$
\left\langle P_{s} f_{x}, f_{y}\right\rangle \leq\left\|P_{t} f_{x}\right\|_{2}\left\|f_{y}\right\|_{2} \leq V\left(x_{0}, r\right)^{1 / 2} V\left(y_{0}, r\right)^{1 / 2}
$$

As $r \geq R / 4$ we have $T>c \Psi(R)$ and so $\Phi(R, T) \leq c^{\prime}$ by Lemma 1.5, and the exponential in (4.23) is of order 1. Adjusting the constant $c_{2}$ we therefore obtain, in both cases,

$$
\left\langle P_{s} f_{x}, f_{y}\right\rangle \leq c_{2} V\left(x_{0}, r\right)^{1 / 2} V\left(y_{0}, r\right)^{1 / 2} \exp \left(-c_{3} \Phi(R, T)\right)
$$

Now set

$$
\widetilde{V}(x, r)=r^{-1} \int_{r}^{2 r} V(x, s) d s .
$$

Then $\widetilde{V}(x, r) \asymp V(x, r)$, and the function $x \rightarrow \widetilde{V}(x, r)$ is continuous. Set

$$
H_{t}(x, y)=c_{4} \widetilde{V}\left(x, \Psi^{-1}(t)\right)^{1 / 2} \widetilde{V}\left(y, \Psi^{-1}(t)\right)^{1 / 2} \exp \left(-c_{3} \Phi(d(x, y), t)\right) .
$$

Then from (4.25) and (4.22) we deduce that $p_{T}^{D}(x, y) \leq H_{T}\left(x_{0}, y_{0}\right)$, for $m \times m$ a.a. $(x, y)$ in a neighbourhood of $\left(x_{0}, y_{0}\right)$. It follows that $p_{T}^{D}(x, y) \leq H_{T}(x, y)$ for $m \times m$ a.a. $(x, y)$.

Now let $D \uparrow \mathcal{X}$; then since $P^{D}$ is ultracontractive it follows from [GT, Theorem 2.12(c)] that

$$
p_{t}(x, y) \leq H_{t}(x, y) \quad \text { for } m \times m \text { a.a. }(x, y) \text { and all } t>0 .
$$

Since the function $H_{t}$ is continuous, by [GT, Theorem 2.12(d)] there exists a properly exceptional set $\mathcal{N}_{1}$ such that if $\mathcal{X}_{1}=\mathcal{X}-\mathcal{N}_{1}$ then

$$
p_{t}(x, y) \leq H_{t}(x, y) \quad \text { for }(x, y) \in \mathcal{X}_{1} \times \mathcal{X}_{1} \text { and all } t>0,
$$

which proves $\mathrm{UHK}(\Psi)$. 


\section{Proof of CSA from upper heat kernel bounds}

In this section we prove the implication $(2) \Rightarrow(1)$ of Theorem 1.12. We assume throughout this section that $\mathcal{X}$ is unbounded, and satisfies $\operatorname{VD}$ and $\operatorname{UHK}(\Psi)$.

We begin by seeing that it is enough to prove (1.17) in a slightly weaker form.

Lemma 5.1. Let $\mathcal{X}$ satisfy VD. Suppose that there exists constants $c_{1}, c_{2}$ such that for all $x \in \mathcal{X}, R>0$ and $r>0$ there exists a cutoff function $\varphi$ for $B(x, R) \subset B(x, r+R)$ such that, if $U=B(x, R+r)-B(x, R)$ and $f \in \mathcal{F}$, then

$$
\int_{U} f^{2} d \Gamma(\varphi, \varphi) \leq c_{1} \int_{U} d \Gamma(f, f)+c_{2} \Psi(r)^{-1} \int_{U} f^{2} d m
$$

Then $\mathcal{X}$ satisfies $\operatorname{CSA}(\Psi)$.

Proof. Let $x \in \mathcal{X}, R>0$ and $r>0$ and $B^{\prime}=B(x, R), B=B(x, R+r)$; we will construct a cutoff function $\varphi$ for $B^{\prime} \subset B$ which satisfies (1.17) with $\theta=c \Psi(r)^{-1}$.

Let $\lambda>0$, and let

$$
s_{n}=c_{0} r e^{-n \lambda / \beta_{2}},
$$

where $c_{0}=c_{0}(\lambda)$ is chosen so that $\sum_{n=1}^{\infty} s_{n}=r$ and $\beta_{2}$ is as in (4.2). Set $r_{0}=0$,

$$
r_{n}=\sum_{k=1}^{n} s_{k}
$$

so that $R<R+r_{1}<R+r_{2}<\cdots<R+r$. Let $B_{n}=B\left(x_{0}, R+r_{n}\right)$, and $U_{n}=B_{n+1}-B_{n}$. By hypothesis there exists a cutoff function $\varphi_{n}$ for $B_{n} \subset B_{n+1}$ satisfying

$$
\int_{U_{n}} f^{2} d \Gamma\left(\varphi_{n}, \varphi_{n}\right) \leq c_{1} \int_{U_{n}} d \Gamma(f, f)+c_{2} \Psi\left(s_{n+1}\right)^{-1} \int_{U_{n}} f^{2} d m
$$

Let $b_{n}=e^{-n \lambda}$ and set

$$
\varphi=\sum_{n=1}^{\infty}\left(b_{n-1}-b_{n}\right) \varphi_{n} .
$$

Then $\varphi=0$ on $B^{c}$, and $\varphi=1$ on $B^{\prime}$, so $\varphi$ is a cutoff function for $B^{\prime} \subset B$. On $U_{n}$ we have

$$
\varphi=\left(b_{n-1}-b_{n}\right) \varphi_{n}+b_{n},
$$

and so $b_{n} \leq \varphi \leq b_{n-1}$ on $U_{n}$. Hence on $U_{n}$

$$
b_{n-1}-b_{n} \leq \frac{\varphi\left(b_{n-1}-b_{n}\right)}{b_{n}}=\left(e^{\lambda}-1\right) \varphi .
$$

Now if $f: B \rightarrow \mathbb{R}$ then by (5.2)

$$
\begin{array}{rl}
\int_{B} f^{2} & d \Gamma(\varphi, \varphi)=\sum_{k=1}^{\infty}\left(b_{k-1}-b_{k}\right)^{2} \int_{U_{k}} f^{2} d \Gamma\left(\varphi_{k}, \varphi_{k}\right) \\
& \leq c_{1} \sum_{k=1}^{\infty}\left(b_{k-1}-b_{k}\right)^{2} \int_{U_{k}} d \Gamma(f, f)+c_{2} \sum_{k=1}^{\infty}\left(b_{k-1}-b_{k}\right)^{2} \Psi\left(s_{k+1}\right)^{-1} \int_{U_{k}} f^{2} d m .
\end{array}
$$


Using (5.4) we have

$$
\begin{aligned}
c_{1} \sum_{k=1}^{\infty}\left(b_{k-1}-b_{k}\right)^{2} \int_{U_{k}} d \Gamma(f, f) & \leq c_{1}\left(e^{\lambda}-1\right)^{2} \sum_{k=1}^{\infty} \int_{U_{k}} \varphi^{2} d \Gamma(f, f) \\
& \leq c_{1}\left(e^{\lambda}-1\right)^{2} \int_{U} \varphi^{2} d \Gamma(f, f) .
\end{aligned}
$$

Now using (4.3) and (5.4)

$$
\frac{\Psi(r)}{\Psi\left(s_{k+1}\right)} \leq\left(\frac{r}{c_{0}(\lambda) r e^{-(k+1) \lambda / \beta_{2}}}\right)^{\beta_{2}}=\frac{e^{\lambda} e^{k \lambda}}{c_{0}(\lambda)^{\beta_{2}}}=\frac{e^{\lambda}\left(e^{\lambda}-1\right)}{c_{0}(\lambda)^{\beta_{2}}\left(b_{k-1}-b_{k}\right)} .
$$

Therefore

$$
\left(b_{k-1}-b_{k}\right) \Psi\left(s_{k+1}\right)^{-1} \leq c_{3}(\lambda) \Psi(r)^{-1}
$$

and hence

$$
\begin{aligned}
c_{2} \sum_{k=1}^{\infty} \int_{U_{k}}\left(b_{k-1}-b_{k}\right)^{2} \Psi\left(s_{k+1}\right)^{-1} f^{2} d m & \leq c_{2} c_{3}(\lambda) \Psi(r)^{-1} \sum_{k=1}^{\infty} \int_{U_{k}} f^{2}\left(b_{k-1}-b_{k}\right) d m \\
& \leq c_{2} c_{3}(\lambda) \Psi(r)^{-1} \int_{U} f^{2}\left(e^{\lambda}-1\right) \varphi d m
\end{aligned}
$$

Thus

$$
\int_{U} f^{2} d \Gamma(\varphi, \varphi) \leq c_{1}\left(e^{\lambda}-1\right)^{2} \int_{U} \varphi^{2} d \Gamma(f, f)+c_{4}(\lambda) \Psi(r)^{-1} \int_{U} f^{2} \varphi d m .
$$

We now choose $\lambda$ so that $c_{1}^{2}\left(e^{\lambda}-1\right)^{2}=1 / 8$ and since $\varphi \leq 1$ we obtain (1.17).

Corollary 5.2. Let $\mathcal{X}$ satisfy VD. Then the condition $\mathrm{CSA}(\Psi)$ is stable.

Proof. Let $\left(\mathcal{E}_{i}, \mathcal{F}\right), i=1,2$ be two Dirichlet forms on $L^{2}(\mathcal{X}, m)$ satisfying the hypothesis of Lemma 1.1, and suppose that $\mathrm{CSA}(\Psi)$ holds for $\mathcal{E}_{1}$. Let $B^{\prime}=B(x, R) \subset B=B(x, R+$ $r$ ), and let $\varphi$ be a cutoff function for $B^{\prime} \subset B$. Then by Lemma 1.1, if $f \in \mathcal{F}, U=B-B^{\prime}$,

$$
\begin{aligned}
\int_{U} f^{2} d \Gamma^{(2)}(\varphi, \varphi) & \leq C \int_{U} f^{2} d \Gamma^{(1)}(\varphi, \varphi) \\
& \leq(C / 8) \int_{U} \varphi^{2} d \Gamma^{(1)}(f, f)+C C_{S} \Psi(r)^{-1} \int_{U} f^{2} d m \\
& \leq\left(C^{2} / 8\right) \int_{U} d \Gamma^{(2)}(f, f)+C C_{S} \Psi(r)^{-1} \int_{U} f^{2} d m .
\end{aligned}
$$

Thus $\left(\mathcal{X}, \mathcal{E}_{2}\right)$ satisfies the condition (5.1) and so by Lemma $5.1 \mathrm{CSA}(\Psi)$ holds for $\mathcal{E}_{2}$.

Now let $\left(X_{t}, t \in \mathbb{R}_{+}, \mathbb{P}^{x}, x \in \mathcal{X}\right)$ be the Hunt process associated with the semigroup $P_{t}$ and Dirichlet form $\mathcal{E}$. Recall the definition of $\mathcal{X}_{0}$ from Section 1 . For a set $D \subset \mathcal{X}$ define the exit time

$$
\tau_{D}=\inf \left\{t>0: X_{t} \in D^{c}\right\}
$$


Lemma 5.3. Suppose $\mathcal{X}$ satisfies $V D$ and $\operatorname{UHK}(\Psi)$. There exists a constant $\varepsilon>0$ such that for all $x \in \mathcal{X}_{0}$ and $r>0$,

$$
\mathbb{P}^{x}\left(\tau_{B(x, r)} \leq \varepsilon \Psi(r)\right) \leq \varepsilon
$$

Proof. In the case $\Psi(r)=r^{\beta}$ this property is denoted $P_{\beta}$ in [GH], and the result follows by [GH, Theorem 2.2]. The general case is similar.

For $D \subset \mathcal{X}, \lambda>0$ set

$$
G_{\lambda}^{D} f(x)=\mathbb{E}^{x} \int_{0}^{\tau_{D}} e^{-\lambda t} f\left(X_{t}\right) d t
$$

Lemma 5.4. Suppose $\mathcal{X}$ satisfies $V D$ and $\operatorname{UHK}(\Psi)$. Let $x_{0} \in \mathcal{X}, r>0, R>0$, and define the annuli $D_{0}=B\left(x_{0}, R+9 r / 10\right)-\bar{B}\left(x_{0}, R+r / 10\right), D_{1}=B\left(x_{0}, R+4 r / 5\right)-\bar{B}\left(x_{0}, R+r / 5\right)$, $D_{2}=B\left(x_{0}, R+3 r / 5\right)-\bar{B}\left(x_{0}, R+2 r / 5\right)$. Let $\lambda=\Psi(r)^{-1}$, and set

$$
h=G_{\lambda}^{D_{0}} 1_{D_{1}} .
$$

Then $h$ has support $\bar{D}_{0}, h \in \mathcal{F}_{D_{0}}$ and satisfies

$$
\begin{aligned}
& h(x) \leq \Psi(r) \quad \text { for all } x \in \mathcal{X}, \\
& h(x) \geq c_{1}^{-1} \Psi(r) \quad \text { for } x \in D_{2} \cap \mathcal{X}_{0} .
\end{aligned}
$$

Proof. That $h \in \mathcal{F}_{D_{0}}$ follows by [FOT, Theorem 4.4.1]. The definition of $h$ implies that $h(x)=0$ for $x \notin \bar{D}_{0}$, and the upper bound on $h$ is elementary, since $h \leq G_{\lambda}^{\mathcal{X}} 1=\lambda^{-1}$.

Now let $\varepsilon>0$ be as in Lemma 5.3. Let $r_{0}=r / 5, x \in D_{2}$, and $B_{1}=B\left(x, r_{0}\right) \subset D_{1}$. Let $s=\varepsilon \Psi\left(r_{0}\right)$, and $\xi_{\lambda}$ be an exponential r.v. independent of $X$ with mean $\lambda^{-1}$. Then

$$
\begin{aligned}
h(x) & \geq \mathbb{E}^{x} \int_{0}^{\xi_{\lambda} \wedge \tau_{D_{0}}} 1_{D_{1}}\left(X_{t}\right) d t \geq \mathbb{E}^{x} \int_{0}^{\xi_{\lambda} \wedge \tau_{B_{1}}} 1_{B_{1}}\left(X_{t}\right) d t \\
& \geq s \mathbb{P}^{x}\left(\xi_{\lambda} \wedge \tau_{B_{1}} \geq s\right)=s \mathbb{P}^{x}\left(\tau_{B_{1}}>s, \xi_{\lambda}>s\right) \\
& =s \mathbb{P}^{x}\left(\tau_{B_{1}}>s\right) \mathbb{P}^{x}\left(\xi_{\lambda}>s\right) \geq s(1-\varepsilon) e^{-\lambda s},
\end{aligned}
$$

which yields (5.11).

Theorem 5.5. Suppose $\mathcal{X}$ satisfies $V D$ and $\operatorname{UHK}(\Psi)$. Then $\mathcal{X}$ satisfies $\mathrm{FK}(\Psi)$ and $\operatorname{CSA}(\Psi)$.

Proof. The proof that $\operatorname{UHK}(\Psi)$ plus VD implies $\mathrm{FK}(\Psi)$ is as in Section 5.5 of [GH], where the case $\Psi(r)=r^{\beta}$ is given.

To prove $\operatorname{CSA}(\Psi)$ we will show that $\mathcal{X}$ satisfies the hypotheses of Lemma 5.1. So let $B^{\prime}=B\left(x_{0}, R\right)$ and $B=B\left(x_{0}, R+r\right)$, and $U=B-B^{\prime}$, and let $D_{i}, h$ be as in Lemma 5.4 . Set

$$
\begin{aligned}
& g(x)=\frac{c_{1} h(x)}{\Psi(r)}, \\
& \varphi(x)= \begin{cases}1 \wedge g(x) & \text { if } x \in B\left(x_{0}, R+r / 2\right)^{c}, \\
1 & \text { if } x \in B\left(x_{0}, R+r / 2\right) .\end{cases}
\end{aligned}
$$


Then by Lemma $5.4 \varphi=0$ on $B^{c}$, and $\varphi=1$ on $B^{\prime}$, so it remains to verify the inequality (5.1).

Let $f \in \mathcal{F}$. Since $g$ is zero outside $U$ we have

$$
\begin{aligned}
\int_{U} f^{2} d \Gamma(\varphi, \varphi) & \leq \int_{U} f^{2} d \Gamma(g, g)=\int_{\mathcal{X}} f^{2} d \Gamma(g, g) \\
& =\int_{\mathcal{X}} d \Gamma\left(f^{2} g, g\right)-2 \int_{\mathcal{X}} f g d \Gamma(f, g) .
\end{aligned}
$$

Now writing $\mathcal{E}_{\lambda}(u, v)=\mathcal{E}(u, v)+\lambda\langle u, v\rangle$,

$$
\begin{aligned}
\int_{\mathcal{X}} d \Gamma\left(f^{2} g, g\right)=\mathcal{E}\left(f^{2} g, g\right) & \leq \mathcal{E}_{\lambda}\left(f^{2} g, g\right) \\
& =c_{1} \Psi(r)^{-1} \mathcal{E}_{\lambda}\left(f^{2} g, G_{\lambda}^{D_{0}} 1_{D_{1}}\right) \\
& =c_{1} \Psi(r)^{-1}\left\langle f^{2} g, 1_{D_{1}}\right\rangle \leq c_{1} \Psi(r)^{-1} \int_{U} f^{2} g d m .
\end{aligned}
$$

Here we used [FOT, Theorem 4.4.1] and the fact that $f^{2} g \in \mathcal{F}_{D_{0}}$ to obtain the third line. By (2.1),

$$
\left|2 \int_{\mathcal{X}} f g d \Gamma(f, g)\right|=\frac{1}{2} \int_{\mathcal{X}} f^{2} d \Gamma(g, g)+2 \int_{\mathcal{X}} g^{2} d \Gamma(f, f) .
$$

Combining (5.15) and (5.16), and using the fact that $g \leq c_{1}$, we obtain

$$
\begin{aligned}
\int_{U} f^{2} d \Gamma(g, g) & \leq 4 \int_{U} g^{2} d \Gamma(f, f)+2 c_{1} \Psi(r)^{-1} \int_{U} g f^{2} d m \\
& \leq 4 c_{1}^{2} \int_{U} d \Gamma(f, f)+2 c_{1}^{2} \Psi(r)^{-1} \int_{U} f^{2} d m .
\end{aligned}
$$

Thus the hypotheses of Lemma 5.1 hold, and so CSA $(\Psi)$ holds.

Remark 5.6. While the proof above is based on the argument in Section 3 of [BBK], it is much simpler, since we do not need to consider the integral over arbitrary balls. Further, the condition $\mathrm{CS}(\Psi)$ requires Hölder continuity of the cutoff function, and this was proved by using a parabolic Harnack inequality, which is equivalent to the full (upper and lower) heat kernel bounds $\operatorname{HK}(\Psi)$. It seems unlikely that the conditions $\operatorname{VD}$ and $\operatorname{UHK}(\Psi)$ are sufficient to ensure the existence of a Hölder continuous cutoff function.

We conclude this section by giving a sketch of the proof that $\operatorname{CSA}(\Psi)$ follows from the condition $\mathrm{CS}(\Psi)$ introduced in [BB3, BBK].

Lemma 5.7. Let $\mathcal{X}$ satisfy VD. Suppose that for every $x \in \mathcal{X}$ and $r>0$ there exists a cutoff function $\varphi$ for $B(x, r) \subset B(x, 2 r)$ such that if $f: B=B(x, 2 r) \rightarrow \mathbb{R}$ then, writing $V=B(x, 2 r)-B(x, r)$,

$$
\int_{V} f^{2} d \Gamma(\varphi, \varphi) \leq c_{1}\left(\int_{V} d \Gamma(f, f)+\Psi(r)^{-1} \int_{V} f^{2} d m\right) .
$$

Then $\mathrm{CSA}(\Psi)$ holds. In particular $\mathrm{CS}(\Psi)$ implies $\operatorname{CSA}(\Psi)$. 
Proof. Let $x_{0} \in \mathcal{X}, R, r>0$, and $B^{\prime}=B\left(x_{0}, R\right)$ and $B=B\left(x_{0}, R+r\right)$, and $U=B-B^{\prime}$. In view of Lemma 5.1 it is enough to prove that there exists $c_{2}<\infty$ such that for $f: U \rightarrow \mathbb{R}$

$$
\int_{U} f^{2} d \Gamma(\varphi, \varphi) \leq c_{2} \int_{U} d \Gamma(f, f)+c_{2} \Psi(r)^{-1} \int_{U} f^{2} d m .
$$

Set $r_{0}=r / 3$, and let $B\left(z_{i}, r_{0}\right)$ be a covering of $B^{\prime}$ by balls such that $B_{i}^{\prime}=B\left(z_{i}, r_{0} / 2\right)$ are disjoint and each $z_{i} \in B^{\prime}$. Then VD implies there exists $M$ such that any ball $B\left(y, r_{0} / 100\right) \subset B$ intersects at most $M$ of the balls $B_{i}$. Let $\varphi_{i}$ be a cutoff function for $B_{i}^{\prime} \subset B_{i}=B\left(z_{i}, 2 r_{0}\right)$ satisfying (5.17). Then

$$
\int_{B_{i}} f^{2} d \Gamma\left(\varphi_{i}, \varphi_{i}\right) \leq c_{1}\left(\int_{B_{i}} d \Gamma(f, f)+\Psi\left(r_{0} / 3\right)^{-1} \int_{B_{i}} f^{2} d m\right) .
$$

Now set $\varphi(x)=\max _{i} \varphi_{i}(x)$. Then $\varphi$ is clearly 1 on $B^{\prime}$ and zero outside $B$.

If $B^{\prime \prime}=B\left(y, r_{0} / 100\right)$ and $B_{i}, i=1, \ldots m$ are the balls which intersect $B^{\prime \prime}$, then

$$
d \Gamma(\varphi, \varphi) \leq \sum_{j=1}^{m} d \Gamma\left(\varphi_{j}, \varphi_{j}\right) .
$$

Thus

$$
\begin{aligned}
\int_{B} f^{2} d \Gamma(\varphi, \varphi) & \leq \sum_{i} \int_{B_{i}} f^{2} d \Gamma\left(\varphi_{i}, \varphi_{i}\right) \\
& \leq \sum_{i} c_{1}\left(\int_{B_{i}} d \Gamma(f, f)+\Psi\left(r_{0} / 3\right)^{-1} \int_{B_{i}} f^{2} d m\right) \\
& \leq c_{1} M\left(\int_{B} d \Gamma(f, f)+\Psi\left(r_{0} / 3\right)^{-1} \int_{B} f^{2} d m\right),
\end{aligned}
$$

proving (5.18).

\section{Stochastic Completeness}

Proof of Theorem 1.16. Following Davies [D, Theorem 7] let $f \geq 0$ be a function with compact support and let $u_{t}=P_{t} f$. We remark that to prove stochastic completeness, by standard density arguments it is sufficient to prove that

$$
\int_{\mathcal{X}} f d m \leq \int_{\mathcal{X}} u_{t} d m \quad \text { for some } t>0 .
$$

Indeed, note that since $P_{t}$ is self-adjoint in $L^{2}(\mathcal{X}, m)$, this implies $\left\langle 1-P_{t} 1, f\right\rangle \leq 0$ and therefore $P_{t} 1=1$ m-a.e.

Let $\left(a_{n}\right)$ be an increasing sequence with $a_{0}=1$, and define $\varphi, b_{n}, b^{*}$ and $C_{0}$ as in (2.2) -(2.4). We assume that $\left(a_{n}\right)$ is chosen so that $b^{*}=1$. Let $t \in(0,1)$. Then

$$
\left\langle f, \varphi_{n}\right\rangle-\left\langle u_{t}, \varphi_{n}\right\rangle=-\int_{0}^{t} \frac{d}{d s}\left\langle u_{s}, \varphi_{n}\right\rangle d s
$$


and

$$
-\frac{d}{d s}\left\langle u_{s}, \varphi_{n}\right\rangle=\mathcal{E}\left(u_{s}, \varphi_{n}\right)=\int_{\mathcal{X}} d \Gamma\left(u_{s}, \varphi_{n}\right) .
$$

So, by Cauchy-Schwarz and Proposition 2.2, and recalling that $t<1$,

$$
\begin{aligned}
\left\langle f, \varphi_{n}\right\rangle-\left\langle u_{t}, \varphi_{n}\right\rangle & =\int_{0}^{t} \int_{\mathcal{X}} \varphi \cdot \varphi^{-1} d \Gamma\left(u_{s}, \varphi_{n}\right) d s \\
& \leq\left(\int_{0}^{t} \int_{\mathcal{X}} \varphi^{2} d \Gamma\left(u_{s}, u_{s}\right) d s\right)^{1 / 2}\left(\int_{0}^{t} \int_{\mathcal{X}} \varphi^{-2} d \Gamma\left(\varphi_{n}, \varphi_{n}\right) d s\right)^{1 / 2} \\
& \leq \sqrt{2}\|f \varphi\|_{2} e^{2 C_{0} t}\left(\sup _{U_{n}} \varphi^{-1}\right)\left(\int_{\mathcal{X}} d \Gamma\left(\varphi_{n}, \varphi_{n}\right)\right)^{1 / 2} .
\end{aligned}
$$

On $U_{n}$ we have $a_{n} \leq \varphi \leq a_{n+1}$, so $\sup _{U_{n}} \varphi^{-1} \leq a_{n}^{-1}$. Using $\operatorname{CSD}\left(D_{n}, D_{n+1}, \theta_{n}\right)$ with $f=1$,

$$
\int_{\mathcal{X}} d \Gamma\left(\varphi_{n}, \varphi_{n}\right)=\int_{U_{n}} d \Gamma\left(\varphi_{n}, \varphi_{n}\right) \leq \theta_{n} m\left(U_{n}\right)
$$

So,

$$
\left\langle f, \varphi_{n}\right\rangle-\left\langle u_{t}, \varphi_{n}\right\rangle \leq \sqrt{2}\|f \varphi\|_{2} \exp \left(2 C_{0} t+\frac{1}{2} \log \left(\theta_{n} m\left(U_{n}\right)\right)-\log \left(a_{n}\right)\right) .
$$

If there exists a subsequence $\left(n_{k}\right)$ such that

$$
\lim _{k \rightarrow \infty}\left(\left\langle f, \varphi_{n_{k}}\right\rangle-\left\langle u_{t}, \varphi_{n_{k}}\right\rangle\right) \leq 0
$$

then, since

$$
\int_{\mathcal{X}} u_{t} d m=\lim _{k} \int_{\mathcal{X}} u_{t} \varphi_{n_{k}} d m
$$

we obtain (6.1) and so deduce stochastic completeness.

(a) If $\theta_{n} \leq c_{1}$ we choose $a_{n}=2^{n}$, so that $b_{n}=b^{*}=1$ and $C_{0}=c_{1}<\infty$. Then (1.20) implies that the right side of (6.2) converges to 0 .

(b) (Recall in this case that $\theta_{n}=c_{0}^{2} n^{2}$.) Let $\alpha>0$, and consider sequences $\left(a_{k}\right)$ such that $C_{0}=C_{0}\left(\left(a_{k}\right)\right)=\alpha^{2}$. We wish $a_{k}$ to be as large as possible given these constraints, and so choose $b_{k}=1 \wedge\left(\alpha / \theta_{k}^{1 / 2}\right)$. Now fix $n \gg 1$, let $m=\lambda \log n$ where $\lambda>0$, and let $\alpha=c_{0} m$. We have

$$
a_{n}=\prod_{j=1}^{n}\left(1+b_{j}\right) \geq \prod_{j=m}^{n}\left(1+\frac{m}{j}\right) .
$$

So since $\log (1+x) \geq \frac{1}{2} x$ for $x \in(0,1)$, for $n$ large enough

$$
\log a_{n} \geq \frac{1}{2} m \sum_{j=m}^{n} j^{-1} \geq \frac{1}{2} \lambda(\log n)(\log n-\log \log n-1) \geq \frac{1}{3} \lambda(\log n)^{2} .
$$

Writing $E(n, \lambda)$ for the term in the exponential in (6.2), if $\log m\left(U_{n}\right) \leq 2 b(\log n)^{2}$ then

$$
\begin{aligned}
E(n, \lambda) & =2 c_{0}^{2} m^{2} t+\frac{1}{2} \log \left(\theta_{n} m\left(U_{n}\right)\right)-\log a_{n} \\
& \leq \frac{1}{2} \log \left(\theta_{n} m\left(U_{n}\right)\right)-(\log n)^{2}\left(\lambda \frac{1}{3}-2 c_{0}^{2} \lambda^{2} t\right) \\
& \leq \log c_{0} n-(\log n)^{2}\left(\frac{\lambda}{3}-b-2 c_{0}^{2} \lambda^{2} t\right) .
\end{aligned}
$$


Choosing $\lambda=9 b$ and $t$ small enough so that $2 c_{0}^{2} \lambda^{2} t \leq b$, it follows that

$$
E(n, \lambda) \leq-b(\log n)^{2}+\log c_{0}^{2} n,
$$

and $(\underline{6.3})$ holds.

Remark 6.1. We have just considered the cases $\theta_{n} \leq c_{1}$ and $\theta_{n}=c_{1} n$, as for our applications these are of most interest. By arguments similar to the above it is straightforward to show that if $\theta_{n}=c_{0}^{2} n^{2 \gamma}$ with $0<\gamma<1$, then stochastic completeness holds provided

$$
\log m\left(U_{n}\right) \leq c(\gamma) n^{1 \wedge(2-2 \gamma)}
$$

We now give some examples of the use of the criterion in Theorem 1.16, and begin by showing that we can recover the result of Davies [D].

Example 6.2. Let $\mathcal{X}$ be a manifold containing a point 0 , and such that there exists $b>0$ such that

$$
m(B(0, r)) \leq e^{b r^{2}}
$$

Let $\left(r_{n}\right)$ be increasing with $\lim r_{n}=\infty$. Set $D_{n}=B\left(0, r_{n}\right)$ and let $U_{n}=D_{n+1}-D_{n}$. Let $\varphi_{n}$ be 'linear' on $U_{n}$, so that

$$
\varphi_{n}(x)=1 \wedge\left(\frac{r_{n+1}-d(0, x)}{r_{n+1}-r_{n}} \vee 0\right), \text { and }\left\|\nabla \varphi_{n}\right\|_{\infty}=\frac{1}{r_{n+1}-r_{n}} .
$$

Letting $\theta_{n}=\left(r_{n+1}-r_{n}\right)^{-2}$, clearly we have

$$
\int_{U_{n}} f^{2} d \Gamma\left(\varphi_{n}, \varphi_{n}\right) \leq \theta_{n} \int_{U_{n}} f^{2} d m,
$$

and so $\operatorname{CSD}\left(D_{n}, D_{n+1}, \theta_{n}\right)$ holds. Let $r_{n}=\log n$, so that $\theta_{n} \sim n^{2}$. Then $m\left(U_{n}\right) \leq$ $m\left(D_{n+1}\right) \leq \exp \left(b(\log (n+1))^{2}\right)$, so (1.21) holds and $\mathcal{X}$ is stochastically complete.

Remark 6.3. Improving the condition $\log V(0, r) \leq b r^{2}$ to $\log V(0, r) \leq r^{2} \log r$ allowed by Theorem 1.15 seems to require more delicate techniques.

\section{The pre-Sierpinski carpet}

In this section we will give an example of an MMD space which is geodesically incomplete but stochastically complete. The example is based on the 'pre-Sierpinski carpet' - see O1.

The standard Sierpinski carpet in $d$ dimensions (with $d \geq 2$ ) can be constructed by an analogue of the construction of the Cantor set. Starting with $F_{0}=[0,1]^{d}$, divide $F_{0}$ into $3^{d}$ subcubes each of side $3^{-1}$, and remove the middle cube; call this set $F_{1}$. Repeating this construction, we obtain a decreasing sequence of compact sets $F_{n}$; the Sierpinski carpet is defined as

$$
F=\cap_{n=0}^{\infty} F_{n} .
$$




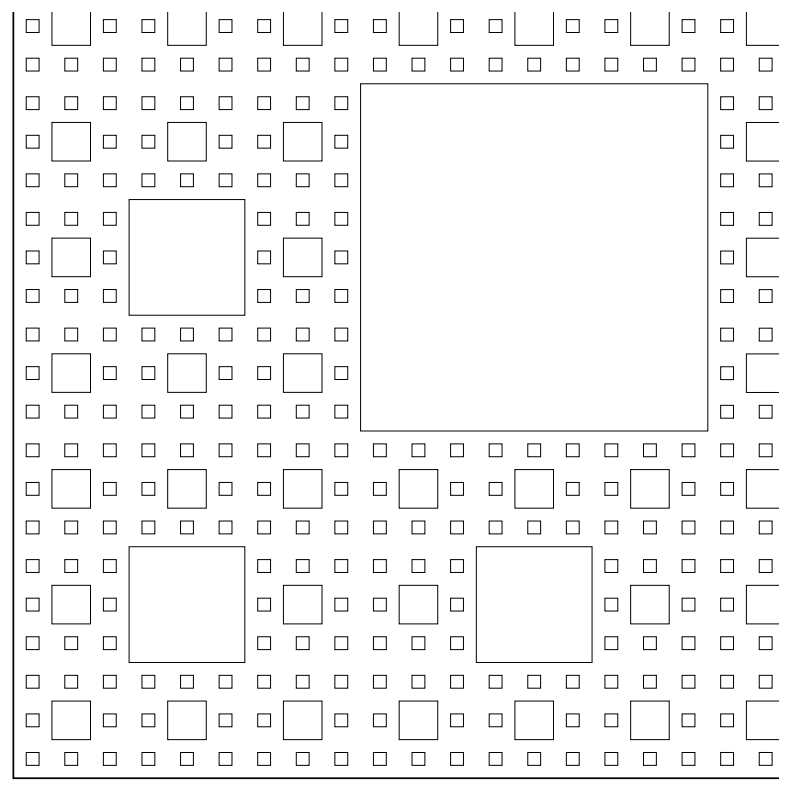

Figure 1: The pre Sierpinski carpet

Let $M_{d}=3^{d}-1$; then $F$ has Hausdorff dimension

$$
d_{f}=\frac{\log M_{d}}{\log 3} .
$$

Note that $F_{n}$ is a union of $M_{d}^{n}$ cubes each of side $3^{-n}$. Let

$$
\widetilde{F}_{n}=3^{n} F_{n}=\left\{3^{n} x: x \in F_{n}\right\}, \quad \widetilde{F}=\cup_{n=0}^{\infty} \widetilde{F}_{n} .
$$

The set $\widetilde{F}$ is the pre-Sierpinski carpet, and is a countable union of copies of the unit cube $[0,1]^{d}$. The interior of $\widetilde{F}$ is a standard open domain in $\mathbb{R}^{d}$, with a Lipschitz boundary. We write $\mathcal{X}=\widetilde{F}$, and will take $d \geq 3$. Let $\mu$ be Lebesgue measure restricted to $\mathcal{X}$.

We summarise some properties of $\mathcal{X}$. Let $d(x, y)$ denote the shortest path distance in $\mathcal{X}$. Then (see [BB1, Lemma 7.3] for the case $d=2$ ) we have

$$
|x-y| \leq d(x, y) \leq c|x-y|, \quad x, y \in \mathcal{X} .
$$

We write $B_{d}(x, r)$ for balls in the metric $d$. Then (see [BB2, Lemma 2.3(e)]) we have

$$
V_{d}(x, r)=\mu\left(B_{d}(x, r)\right) \asymp \begin{cases}r^{d}, & 0 \leq r \leq 1, \\ r^{d_{f}}, & r>1 .\end{cases}
$$

In particular $\mathcal{X}$ satisfies VD. 
Now set

$$
\mathcal{E}(f, f)=\int_{\mathcal{X}}|\nabla f|^{2} d \mu, \quad f \in H^{1}(\mathcal{X})
$$

where $H^{1}=H^{1}(\mathcal{X})$ denotes the set of functions $f$ for which $\int_{\mathcal{X}} f^{2} d x+\mathcal{E}(f, f)<\infty$. Then $\left(\mathcal{E}, H^{1}\right)$ is a regular local Dirichlet form on $L^{2}(\mathcal{X}, \mu)$. The associated Hunt process $W=\left(W_{t}, t \geq 0, \mathbb{P}^{x}, x \in \mathcal{X}\right)$ is Brownian motion in $\mathcal{X}$ with normal reflection on the boundary $\partial \mathcal{X}$. For the existence and uniqueness in law of this process we refer to $[\mathrm{BH}]$. The process is reversible with respect to $\mu$, and its generator is given by the Neumann Laplacian $\Delta$ on $\mathcal{X}$.

Let $p_{t}(x, y)$ denote the heat kernel associated with $W$. Many of the properties of $W$ and $p_{t}$ can be summarised by two indices. The first is $d_{f}$, the Hausdorff dimension of the space $F$. The second, denoted $d_{w}$, and called the walk dimension, gives the long range space-time scaling on $\mathcal{X}$. For Sierpinski carpets in $d \geq 3$ this satisfies $2<d_{w}<d_{f}$ - see [BB2, Section 5]. Let $\Psi=\Psi_{2, d_{w}}$ be as defined in (1.3).

Theorem 7.1. (a) $(\mathcal{X}, \mathcal{E})$ satisfies $\operatorname{HK}(\Psi)$.

(b) $W$ has a Greens function $g(x, y)$ such that there exist positive constants $c_{1}-c_{4}$ such that

$$
\begin{aligned}
c_{1}|x-y|^{2-d} & \leq g(x, y) \leq c_{2}|x-y|^{2-d} \quad \text { if }|x-y| \leq 1, \\
c_{3}|x-y|^{d_{w}-d_{f}} & \leq g(x, y) \leq c_{4}|x-y|^{d_{w}-d_{f}} \quad \text { if }|x-y|>1 .
\end{aligned}
$$

(c) The conditions $\mathrm{CS}(\Psi)$ and $\mathrm{CSA}(\Psi)$ hold for $(\mathcal{X}, \mathcal{E})$.

Proof. (a) is proved in [BB2, Theorem 6.9], and (b) in [BB2, Corollary 6.10]. That $\mathrm{CS}(\Psi)$ holds follows from [BBK]. CSA $(\Psi)$ then follows by Lemma 5.7, or alternatively by Theorem 1.12 .

Let $a(x)>0, x \in \mathcal{X}$ be a real-valued function on $\mathcal{X}$. Then, we define the additive functional

$$
A_{t}=\int_{0}^{t} \frac{1}{a\left(W_{s}\right)} d s
$$

and the time-changed process $Y=Y^{(a)}$ by

$$
Y_{t}=W_{\tau_{t}}, \quad t \geq 0,
$$

where $\left(\tau_{t}\right)$ denotes the inverse of $\left(A_{t}\right)$. The process $Y$ is symmetric with reversible measure $m(d x)=m_{a}(d x)=a^{-1}(x) \mu(d x)$ and its generator $\mathcal{L}_{a}$ satisfies

$$
\int g \mathcal{L}_{a} f a^{-1} d \mu=\left\langle\mathcal{L}_{a} f, g\right\rangle_{L^{2}\left(m_{a}\right)}=-\mathcal{E}(f, g)=-\int \nabla f \cdot \nabla g d \mu=\int(\Delta f) g d \mu,
$$

so that

$$
\mathcal{L}_{a} f=a \Delta f
$$


The Dirichlet form associated with $Y$ is the form $\left(\mathcal{E}, \mathcal{D}_{a}\right)$ on the base space $L^{2}\left(\mathcal{X}, m_{a}\right)$. Here $\mathcal{D}_{a}$ is the closure of $C_{0}^{1}(\mathcal{X})$ with respect to $N_{a}(f)=\mathcal{E}(f, f)+\|f\|_{L^{2}\left(m_{a}\right)}^{2}$. We refer to this form as $\mathcal{E}_{a}$ for short. Recall from (1.18) the definition of the intrinsic metric $\varrho_{a}$ associated with $\mathcal{E}_{a}$; we have

$$
\varrho_{a}(x, y)=\sup \left\{u(x)-u(y): u \in \mathcal{M}_{a},\right\},
$$

where

$$
\mathcal{M}_{a}=\left\{u \in \mathcal{D}_{a} \cap C(\mathcal{X}):|\nabla u|^{2} \leq a^{-1}\right\}
$$

Let $p>0$. We now just consider the case

$$
a(x)=1 \vee d(0, x)^{p} .
$$

The main result of this section is the following. Recall that we have $d \geq 3$, and that $2<d_{w}<d_{f}$.

Theorem 7.2. (i) The process $Y=Y^{(p)}$ is stochastically complete if and only if $p \leq d_{w}$. (ii) On the other hand, (VGC) holds if and only if $p \leq 2$ or $p>d_{f}$. In particular, for $p \in\left(2, d_{w}\right)$ the process $Y$ is stochastically complete but (VGC) fails.

We begin by relating the metrics $\varrho_{a}$ and $d$ on $\mathcal{X}$.

Lemma 7.3. Let $R>0$, and $x \in \partial B_{d}(0, R), y \in \partial B_{d}(0,2 R)$. Then

$$
\varrho_{a}(x, y) \asymp R^{1-p / 2} \text {, if } R \geq 1,
$$

while $\varrho_{a}(x, y) \asymp R$ if $R \in[0,1]$.

Proof. By Theorem 4.1 in Chapter 5 of St2] we have

$$
\varrho_{a}(0, x)=\inf _{\gamma} \int_{0}^{1}|\dot{\gamma}| \frac{1}{\sqrt{a(\gamma(s))}} d s .
$$

If $\gamma$ is any path in $B_{d}(0,2 R)-B_{d}(0, R)$ then

$$
\int_{0}^{1}|\dot{\gamma}| \frac{1}{\sqrt{a(\gamma(s))}} d s \asymp R^{-p / 2} \int \dot{\gamma}=R^{-p / 2}|\gamma|,
$$

where $|\gamma|$ denotes the length of $\gamma$. It follows that $\varrho_{a}(x, y) \geq c R^{1-p / 2}$.

For the upper bound, the geometry of the pre-carpet implies that if $C>1$ is large enough then we can find a path $\gamma_{1}$ between $x$ and $y$ which lies inside $B_{d}(0, C R)-B_{d}(0, R)$ and has length less than $c_{1} R$. Therefore $\varrho_{a}(x, y) \leq c_{2} R^{1-p / 2}$.

Proposition 7.4. The metric $\varrho_{a}$ and measure $m_{a}$ satisfy the following.

(i) $\varrho_{a}(0, \infty)=\infty$ if and only if $p \leq 2$. In particular, $\left(\mathcal{X}, \varrho_{a}\right)$ is not geodesically complete when $p>2$.

(ii) $m_{a}(\mathcal{X})=\infty$ if and only if $p \leq d_{f}$. 
Proof. (i) Let $x_{k}=\left(2^{k}, 0, \ldots, 0\right)$ be the points on $\partial B_{d}\left(0,2^{k}\right)$. Then by Lemma 7.3 we have $\varrho_{a}\left(x_{k}, x_{k+1}\right) \asymp 2^{k(1-p / 2)}$. If $p \leq 2$ the sum $\sum_{k} \varrho_{a}\left(x_{k}, x_{k+1}\right)$ diverges, and hence $\varrho_{a}\left(0, x_{k}\right) \rightarrow \infty$, while if $p>2$ then $\lim _{k} \varrho_{a}\left(0, x_{k}\right) \leq C_{1}<\infty$; (i) then follows.

(ii) By (7.3)

$$
\begin{aligned}
m_{a}(\mathcal{X}) & =\sum_{k} m_{a}\left(B_{d}\left(0,2^{k+1}\right)-B_{d}\left(0,2^{k}\right)\right) \\
& \asymp \sum_{k} 2^{-k p} \mu\left(B_{d}\left(0,2^{k+1}\right)-B_{d}\left(0,2^{k}\right)\right) \asymp \sum_{k} 2^{-k p} 2^{k d_{f}},
\end{aligned}
$$

which is infinite if and only if $p \leq d_{f}$.

We now look at (VGC) for the metric measure space $\left(\mathcal{X}, \varrho_{a}, m_{a}\right)$. We set $h^{-1}(r):=$ $\int_{0}^{r}(1 \vee s)^{-p / 2} d s, r \geq 0$, so that $\varrho_{a}(0, x) \asymp h^{-1}(|x|)$ if $|x| \geq 1$. Further, let $h(r):=\inf \{s:$ $\left.h^{-1}(s)>r\right\}$ be the right-continuous inverse. In particular,

$$
B_{d}\left(0, h\left(c_{2} r\right)\right) \subseteq B_{\varrho_{a}}(0, r) \subseteq B_{d}\left(0, h\left(c_{1} r\right)\right), \quad r>1 .
$$

Moreover, if $p \leq 2$ we have $\lim _{r \rightarrow \infty} h^{-1}(r)=\infty$, so $h(r)<\infty$ for all $r$. On the other hand, if $p>2$ we have that $R_{0}:=\lim _{r \rightarrow \infty} h^{-1}(r)<\infty$, thus $h(r)=\infty$ and $B_{\varrho_{a}}(0, r)=F$ for $r \geq R_{0}$.

Lemma 7.5. Let $p \leq 2<d_{f}$. There exist positive constants $c_{1}-c_{5}$ and $r_{0}$ such that for all $r>r_{0}$

$$
c_{1} h\left(c_{2} r\right)^{d_{f}-p} \leq m_{a}\left(B_{\varrho_{a}}(0, r)\right) \leq c_{3} h\left(c_{4} r\right)^{c_{5}\left(d_{f}-p\right)} .
$$

Proof. The lower bound is immediate from (7.3) as

$$
\begin{aligned}
m_{a}\left(B_{\varrho_{a}}(0, r)\right) & \geq \int_{B_{d}(0, h(c r))} \frac{1}{a(x)} \mu(d x) \\
& \geq \mu\left(B_{d}(0,1)\right)+h(r)^{-p}\left(\mu\left(B_{d}(0, h(c r))\right)-\mu\left(B_{d}(0,1)\right)\right) \\
& \geq c+c h(c r)^{d_{f}-p}
\end{aligned}
$$

for $r$ sufficiently large, where we used the fact that $h$ is increasing.

To prove the upper bound note that for $k \geq 0$ we have $a(x) \geq 2^{k p}$ on the set $U_{k}=$ $B_{d}\left(0,2^{k+1}\right)-B_{d}\left(0,2^{k}\right)$. Let $k_{0}(r)=\min \left\{k: 2^{k} \geq h(c r)\right\}$. Then, for all $r$ large enough we have again by (7.3)

$$
\begin{aligned}
m_{a}\left(B_{\varrho_{a}}(0, r)\right) & \leq \int_{B_{d}(0, h(c r))} \frac{1}{a(x)} \mu(d x) \leq \mu\left(B_{d}(0,1)\right)+c \sum_{k=0}^{k_{0}(r)} m_{a}\left(U_{k}\right) \\
& \leq c+c \sum_{k=0}^{k_{0}(r)} 2^{k\left(d_{f}-p\right)} \leq c h(c r)^{c\left(d_{f}-p\right)} .
\end{aligned}
$$


Proof of Theorem 7.2

(i) First let $p \leq d_{w}$. Let $R>1, R_{n}=R^{n}, D_{n}:=B_{d}\left(0, R_{n}\right)$ and $U_{n}=D_{n+1}-D_{n}$. Thus

$$
m_{a}\left(U_{n}\right) \asymp R_{n}^{-p} \mu\left(U_{n}\right) \asymp R_{n}^{d_{f}-p} .
$$

By Theorem 7.1 we have $\operatorname{CSA}(\Psi)$ for the space $(\mathcal{X}, d, \mathcal{E}, \mu)$. So there exists a cutoff function $\varphi_{n}$ for $D_{n} \subset D_{n+1}$ such that if $f: U_{n} \rightarrow \mathbb{R}$ then

$$
\begin{aligned}
\int_{U_{n}} f^{2} d \Gamma\left(\varphi_{n}, \varphi_{n}\right) & \leq \frac{1}{8} \int_{U_{n}} \varphi_{n}^{2} d \Gamma(f, f)+c_{0} R_{n}^{-d_{w}} \int_{U_{n}} f^{2} d \mu \\
& \leq \frac{1}{8} \int_{U_{n}} \varphi_{n}^{2} d \Gamma(f, f)+c_{1} R_{n}^{p-d_{w}} \int_{U_{n}} f^{2} d m_{a} .
\end{aligned}
$$

Thus in the space $\left(\mathcal{X}, \varrho_{a}, m_{a}\right), \operatorname{CSD}\left(D_{n}, D_{n+1}, \theta_{n}\right)$ holds with $\theta_{n}=c_{1} R_{n}^{p-d_{w}}$. As $p \leq d_{w}$ we have $\theta_{n} \leq c_{1}$ and hence by Theorem 1.16(a) stochastic completeness holds provided (1.20) holds. However,

$$
\frac{\theta_{n} m_{a}\left(U_{n}\right)}{4^{n}} \leq c_{1} 4^{-n} R_{n}^{-d_{w}+p} R_{n}^{d_{f}-p}=c_{1}\left(R^{d_{f}-d_{w}} / 4\right)^{n},
$$

and taking $R$ small enough so that $R^{d_{f}-d_{w}}<4$ it follows that stochastic completeness holds.

Now we consider the case $p>d_{w}$. Since the process $W$ is stochastically complete, from the definition of stochastic completeness it is immediate that $Y$ is stochastically complete if and only if $A_{\infty}=\infty \mathbb{P}^{x}$-a.s. for any $x$. Note that $A_{\infty}=\infty$ is a tail event, i.e. it is in $\sigma\left(W_{s}, s \geq t\right)$ for all $t$, so $\mathbb{P}^{x}\left[A_{\infty}=\infty\right]$ is either 0 or 1 for all $x$ (cf. Theorem 8.7 in [BB2]).

Let $D_{0}=B_{d}(0,1)$ and for $n \geq 1$ set $D_{n}=B_{d}\left(0,2^{n}\right)-B_{d}\left(0,2^{n-1}\right)$. Then using the bounds for the Green kernel $g(x, y)$ of $W$ in Theorem 7.1 (b),

$$
\begin{aligned}
\mathbb{E}^{0} A_{\infty} & =\int_{\mathcal{X}} a(x)^{-1} g(0, x) \mu(d x) \\
& \leq c \int_{B_{d}(0,1)}|x|^{2-d} d x+c \sum_{n=1}^{\infty} \int_{D_{n}} d(0, x)^{-p+d_{w}-d_{f}} d x \\
& \leq c+c \sum_{n=1}^{\infty} 2^{n\left(-p+d_{w}-d_{f}\right)} 2^{n d_{f}} \leq c+c \sum_{n=1}^{\infty} 2^{n\left(d_{w}-p\right)}<\infty,
\end{aligned}
$$

Hence, $A_{\infty}<\infty \mathbb{P}^{0}$-a.s., and so $Y$ is stochastically incomplete.

(ii) Let us first consider the case $p<2$. Then, $d_{f}-p>0$ and $h(r) \asymp r^{\gamma}$ for large $r$ with $\gamma:=(1-p / 2)^{-1}$. Hence, we use (17.3) to obtain

$$
\int_{1}^{\infty} \frac{r}{\log m_{a}\left(B_{\varrho a}(0, r)\right)} d r \geq \int_{1}^{\infty} \frac{r}{c_{1}+c_{2} \log r} d r=\infty .
$$

If $p=2, h(r)=e^{r-1}$, so

$$
\int_{1}^{\infty} \frac{r}{\log m_{a}\left(B_{\varrho_{a}}(0, r)\right)} d r \geq \int_{1}^{\infty} \frac{r}{c_{1}+c_{2}(r-1)} d r=\infty .
$$


Finally, in the case $p>2$ we have that $R_{0}:=\lim _{r \rightarrow \infty} h^{-1}(r)<\infty$, thus $h(r)=\infty$ and $B_{\varrho_{a}}(0, r)=\mathcal{X}$ for $r \geq R_{0}$. In particular, by Proposition 7.4 ii) we get for such $r$ that $m_{a}\left(B_{\varrho_{a}}(0, r)\right)=m_{a}(\mathcal{X})=\infty$ if and only $p \leq d_{f}$. Hence,

$$
\int_{1}^{\infty} \frac{r}{\log m_{a}\left(B_{\varrho_{a}}(0, r)\right)} d r \begin{cases}<\infty & \text { if } p \leq d_{f} \\ =\infty & \text { if } p>d_{f} .\end{cases}
$$

\section{References}

[Ar] D.G. Aronson, Bounds for the fundamental solution of a parabolic equation, Bull. Amer. Math. Soc. 73 (1967), pp.890-896.

[Ba1] M.T. Barlow. Diffusions on fractals. In: Lectures on Probability Theory and Statistics, Ecole d'Éte de Probabilités de Saint-Flour XXV - 1995, 1-121. Lect. Notes Math. 1690, Springer 1998.

[BB1] M.T. Barlow, R.F. Bass. Transition densities for Brownian Motion on the Sierpinski Carpet. Probab. Theory Relat. Fields 91(1992), 307-330.

[BB2] M.T. Barlow, R.F. Bass. Brownian Motion and Harmonic Analysis on Sierpinski Carpets. Canad. J. Math. Vol. 51 (4) (1999), 673-744.

[BB3] M.T. Barlow, R.F. Bass. Stability of parabolic Harnack inequalities. Trans. Amer. Math. Soc. 356 (2003) no. 4, 1501-1533.

[BBK] M.T. Barlow, R.F. Bass and T. Kumagai. Stability of parabolic Harnack inequalities on metric measure spaces. J. Math. Soc. Japan (2) 58 (2006), 485-519.

[Bas] R.F. Bass. A stability theorem for elliptic Harnack inequalities, J. Europ. Math. Soc., to appear.

[BH] R.F. Bass and E. Hsu. Some potential theory for reflecting Brownian motion in Hölder and Lipschitz domains. Ann. Probab. 19 (1991), no. 2, 486-508.

[CG] T. Coulhon, A. Grigoryan. A. Random walks on graphs with regular volume growth. Geom. Funct. Anal. 8 (1998), no. 4, 656701.

[D] E.B. Davies. Heat kernel bounds, conservation of probability and the Feller property. J. d'Analyse Math. 58, 99-119. (1992)

[FS] E.B. Fabes, D.W. Stroock. A new proof of Moser's parabolic Harnack inequality using the old ideas of Nash. Arch. Mech. Rat. Anal. 96 (1986), 327-338.

[FOT] M. Fukushima, Y. Oshima, M. Takeda. Dirichlet Forms and Symmetric Markov Processes. de Gruyter, Berlin, 1994. 
[Gr0] A. Grigor'yan. The heat equation on noncompact Riemannian manifolds. (in Russian) Matem. Sbornik. 182 (1991), 55-87. (English transl.) Math. USSR Sbornik 72 (1992), 47-77.

[Gr1] A.A. Grigor'yan. On stochastically complete manifolds. Soviet Math. Dokl. 34 (1987), 310-313.

[Gr2] A.A. Grigor'yan. Analytic and geometric background of recurrence and nonexplosion of the Brownian motion on Riemannian manifolds. Bull. Amer. Math. Soc. (N.S.) 36 (1999), 135-249.

[Gr3] A. Grigor'yan. Heat kernel upper bounds on a complete non-compact manifold. Revista Math. Iberoamericana 10 (1994) no.2, 395-452

[GH] A. Grigor'yan, J. Hu. Upper bounds of heat kernels on doubling spaces. Preprint 2010.

[GT] A. Grigor'yan, A. Telcs. Two-sided estimates of heat kernels on metric measure spaces. Ann. Prob. 2011.

[Hi] M. Hino. On singularity of energy measures on self-similar sets. Prob. Th. Rel. Fields, 132 (2005), 265-290.

[LJ] Y. Le Jan. Mesures associees a une forme de Dirichlet. Applications. Bull. Soc. Math. France 106 (1978), no. 1, 61-112.

[LY] P. Li and S.-T. Yau, On the parabolic kernel of the Schrödinger operator Acta Math. 156 (1986), pp.153-201.

[Kan] M. Kanai. Rough isometries and combinatorial approximations of geometries of non-compact riemannian manifolds. J. Math. Soc. Japan 37 (1985), 391-413.

[Mos] U. Mosco. Composite media and asymptotic Dirichlet forms. J. Funct. Anal. 123 (1994), no. 2, 368-421

[Mo1] J. Moser. On Harnack's inequality for elliptic differential equations. Comm. Pure Appl. Math. 14, (1961) 577-591.

[O1] H. Osada: Isoperimetric dimension and estimates of heat kernels of pre-Sierpinski carpets. Probab. Th. Rel. Fields 86, 469-490 (1990).

[SC1] L. Saloff-Coste. A note on Poincaré, Sobolev, and Harnack inequalities. Inter. Math. Res. Notices 2 (1992), 27-38.

[SCS] L. Saloff-Coste, D.W. Stroock. Opérateurs uniformément sous-elliptiques sur les groupes de Lie. J. Funct. Anal. 98 (1991) 97-121.

[St1] K.-T. Sturm. Analysis on local Dirichlet spaces I. Recurrence, conservativeness and $L^{p}$-Liouville properties. J. reine angew. Math. 456 (1994), 173-196. 
[St2] K.-T. Sturm. The geometric aspect of Dirichlet forms. In: J. Jost, W. Kendall, U. Mosco, M. Röckner, K.-T. Sturm. New Directions in Dirichlet Forms. AMS/IP Studies in Advanced Mathematics 8 (1998), American Mathematical Society, Providence, RI; International Press.

SA: Institut für Angewandte Mathematik

Rheinische Friedrich-Wilhelms-Universität Bonn

Endenicher Allee 60, 53115 Bonn, Germany.

andres@iam.uni-bonn.de

MB: Department of Mathematics, University of British Columbia, Vancouver, B.C. V6T 1Z2, Canada.

barlow@math.ubc.ca 